\title{
Hybrid frequency allocation scheme for capacity improvement in densely deployed small cells
}

\author{
Qixun Zhang ${ }^{*}$, Zhiyong Feng, Yue Zhang and Tuo Yang
}

\begin{abstract}
Driven by the demands for better user experience and high data-rate service, there is an ever increasing trend for capacity enhancement for wireless networks. The densely deployed small cells is a promising solution which can provide a huge capacity gain and improve the user experience with high data-rate services. Due to the random deployed manner of the customers, small cells should have automatic parameters an tuning and optimization abilities to realize the self-deployment in heterogeneous networks. Therefore, a novel dynamic hybrid frequency allocation scheme is proposed by applying both inner and outer circle regions for different frequency allocation schemes for small cells, which can improve the capacity and minimize interference among different hierarchical networks. Closed-form solutions are achieved for proposed downlink capacity model and key parameters affecting the capacity are also analyzed thoroughly. Furthermore, an optimal geographic region division scheme is designed and the optimal square zone length is theoretically obtained for an efficient information delivery among small cells. Numerical results verify that the capacity can be improved with the appropriate small cell deployment using proposed novel schemes.
\end{abstract}

Keywords: Small cell; Self-deployment; Femtocell; Heterogeneous networks

\section{Introduction}

According to recent studies, $50 \%$ of phone calls and $70 \%$ of data services will take place indoors in the coming years [1]. Moreover, over two thirds of voice services and about $90 \%$ of data services will take place indoors [2]. Studies also show that more than $45 \%$ of households and $30 \%$ of businesses users experienced inadequate indoor coverage [3], leading to the poor quality-of-service (QoS). Furthermore, Cisco forecasts that the global mobile data traffic grew $81 \%$ in 2013 and smarter mobile devices are increasing fast in [4]. Moreover, the uneven traffic distribution leads to new challenges for the coverage optimization and capacity enhancement. But traditional wireless network planning and optimization techniques cannot guarantee both a wide range coverage and an effective service quality indoors, leading to the expansion of network capacity as a fundamental problem for mobile network operators.

\section{*Correspondence: zhangqixun@bupt.edu.cn}

Key Laboratory of Universal Wireless Communications, Ministry of Education, Information and Telecommunication Engineering of Beijing University of Posts and Telecommunications (BUPT), Xitucheng Road, Beijing, Haidian District, 100876, P.R. China
Traditional network capacity improvement techniques such as cell splitting and adding more spectrum cannot meet the demands of capacity enhancement, uneven traffic distribution, and dynamic changing services. Besides, due to the building blocking, signal propagation loss, and reflection effect, capacity holes in hot spots and deteriorated user experiences are still big challenges [5]. Therefore, how to make full use of heterogeneous networks resources, how to decrease the complexity of resource management, and how to improve network capacity and user experiences indoors are still problems unsolved. Traditional network planning and optimization techniques face the problems of high implementation cost, long deployment period, and complex optimization process. Therefore, the self-deployed small cells, such as femtocell, picocell, and microcell, for indoor capacity enhancement and coverage optimization are proposed recently and considered as one of the efficient solutions in [6, 7].

As one of the efficient capacity enhancement techniques, small cells are designed to operate on the licensed bands for both indoor and outdoor scenarios in [3] and [8]. Moreover, small cells can provide a fast, flexible, and

\section{是 Springer}

(c) 2015 Zhang et al. This is an Open Access article distributed under the terms of the Creative Commons Attribution License (http://creativecommons.org/licenses/by/4.0), which permits unrestricted use, distribution, and reproduction in any medium, provided the original work is properly credited. 
cost-efficient solution for existing cellular networks in [9]. Small cells include femtocells, picocells, microcells, and metrocells [10]. Small cells can be easily deployed in hotspots and indoor scenarios to improve the capacity and user experience. However, challenges of small cell deployment still exist, including interference analysis among different heterogeneous networks, frequency allocation scheme, and access mode selection for small cells.

In the literature, existing research works on capacity analysis of small cells are studied by considering different frequency allocation schemes and interference issues. In terms of uplink capacity analysis in hierarchical networks, both the closed subscriber group (CSG) access mode and open access mode are considered in orthogonal frequency division multiple access (OFDMA) and time division multiple access (TDMA)-based femtocell networks in [8]. Furthermore, the macrocell users' density is proposed as a key factor to the optimal selection of CSG and open access modes, where the open access mode is preferred when macrocell users' density is small. Moreover, open access mode is applied in code division multiple access (CDMA)-based femtocell networks in [11]. A distributed orthogonal frequency allocation scheme is proposed by using the optimal frequency allocation ratio between femtocell and macrocell networks in [12] to minimize the interference among different layers in hierarchical networks. In [13], both the joint and disjoint subchannel allocation schemes are proposed for two-tier networks with quality-of-service constraints in terms of success probabilities and per-tier minimum rates. Considering the frequency allocation in hierarchical networks, a hybrid orthogonal frequency allocation scheme is proposed in [14], which considers about the distance between femtocell and macrocell and the interference constraint area (ILCA) factor.

In terms of interference problems among OFDMAbased hierarchical networks, an intelligent self-organized femtocell network based on real-time multi-agent reinforce learning technology is proposed in [15] by using the accumulated interference from different femtocell networks to solve the interference problems. Moreover, a docition-based real-time scheme is also proposed in [15] to improve the learning ability and accuracy of Q-learning process, which solves the unstable decisionmaking problem and the drawbacks of complex learning process and slow learning speed. Considering the uplink capacity and interference cancelation problems in CDMA-based hierarchical networks, the uplink capacity and time-hop (TH)-based CDMA interference cancelation technology are proposed and analyzed with the outage probability constraints of macrocell and femtocell in co-channel frequency deployment scenario [16]. Furthermore, in OFDMA-based hierarchical networks, selfconfiguration- and self-optimization-based interference cancelation schemes are proposed to solve the interference problems in co-channel and orthogonal frequency allocation schemes in [17] and [18].

Considering the scarcity of spectrum resources, the small cell sharing the same spectrum with macrocells is an acceptable and efficient way of improving the network capacity. Most recent studies on the frequency allocation and capacity analysis for small cells are using co-channel and orthogonal frequency allocation schemes in the single cell scenario, which apply the interference cancelation methods to improve the network capacity. However, considering that small cells are typically deployed randomly by users without network planning, it is important that the intelligent self-organizing techniques should be applied in small cells. Besides, more and more researchers are paying attention to adding cognitive technologies in femtocell networks in [19-21]. But how to add intelligent abilities for small cell optimization is a new challenge. Therefore, this paper proposes a hybrid frequency allocation scheme to increase the downlink capacity in densely deployed small cell networks. First, the downlink network capacity of small cell networks is analyzed by using different frequency allocation schemes, which includes orthogonal, co-channel, and hybrid frequency allocation schemes. Both the density of femtocells and interference constraints to guarantee the quality of macrocells are considered and verified with theoretical results in this paper. The proposed hybrid frequency allocation scheme utilizes different frequency allocation schemes for the inner and outer circle regions to improve the network capacity. By adding the geographic region cognition ability in densely deployed small cells, an optimal geographic region division scheme is designed and the optimal square zone length is theoretically obtained for an efficient information delivery among small cells via cognitive pilot channel (CPC) [22, 23]. Numerical results verify that the capacity can be improved with the appropriate small cell deployment using proposed novel schemes.

The remainder of this paper is organized as follows. The system model and scenarios for densely deployed small cell networks are proposed in Section 2. The downlink capacity of heterogeneous networks using different frequency allocation schemes are proposed and analyzed with theoretical proofs in Section 3. Optimal geographic region division scheme is described in Section 4. The self-deployment procedure of hybrid frequency allocation scheme is proposed in Section 5. Numerical results are presented and verified in Section 6. Finally, Section 7 concludes this paper.

\section{System model and scenario}

A typical architecture of heterogeneous networks with macrocell base stations (MBSs) and small cells is overlapped as depicted in Fig. 1. In this paper, three different 


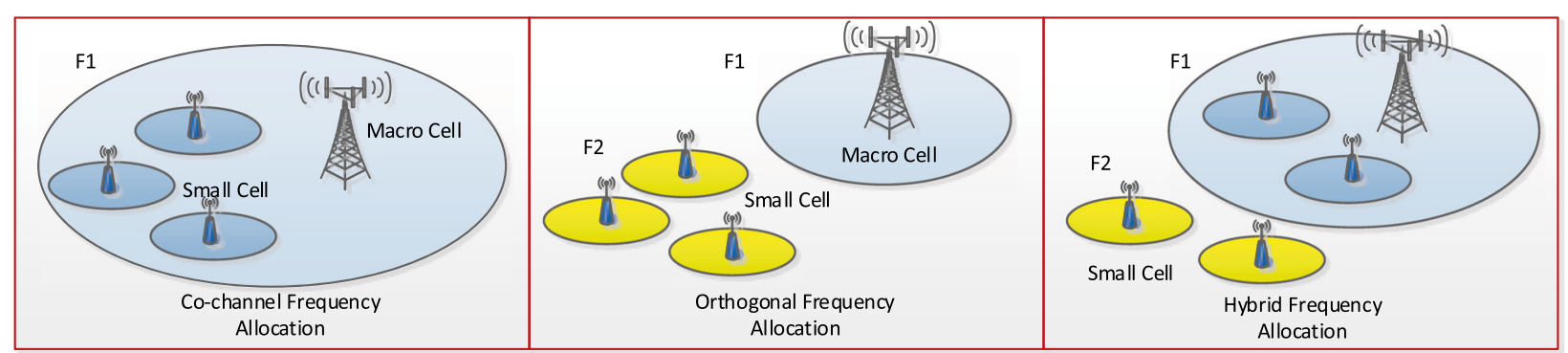

Fig. 1 Small cell network architecture and three frequency allocations schemes

frequency allocation schemes, including orthogonal, cochannel, and hybrid frequency allocation schemes, are described and compared in this paper, considering the impacts on downlink capacity and interference in heterogeneous networks.

Orthogonal frequency allocation Different frequency bands are assigned to macrocell layer and small cell layer separately. Although the orthogonal frequency allocation can reduce cross-layer interference, its disadvantage is a much lower spectral efficiency. Furthermore, due to the scarce of available spectrum, interference among small cells is a big problem, especially in the densely deployed small cell scenario.

Co-channel frequency allocation Both macrocells and small cells share the same frequency. Due to its low transmit power and small coverage area, the inter-cell interference between macrocell and small cell can be avoided by using the spatial isolation, which can improve the network capacity. However, when the small cells are densely deployed, the interference issues cannot be solved only by using the spatial isolation scheme, which will decrease the network capacity.

Hybrid frequency allocation The coverage area of macrocell is divided into inner and outer circle regions. Small cells located within the inner circle region apply the orthogonal frequency allocation scheme, in order to decrease the interference to macrocells. When small cells are in the outer circle region, the co-channel frequency allocation scheme can be applied to improve the network capacity by utilizing the spatial isolation to minimize the interference.

In this paper, it is assumed that the coverage area of MBS is modeled as a hexagon with radius $R_{m}$, and the coverage area is represented by $H$ as $|H|=3 \sqrt{3} R_{m}^{2}$. The distribution of macrocell users (MUE) is a Poisson point process (PPP) [24-26] with density $\lambda_{\text {MUE }}$. The position of MUE is depicted as $\Phi_{\text {MUE }}=\left\{X_{i}\right\}$. Each MBS has six neighbors denoted by $\operatorname{MBS}_{i}(i=1,2, \ldots, 6)$. The distribution of small cells, such as femto base station (FBS) is modeled as a PPP [24-26] with density $\lambda_{\mathrm{FBS}}$. The position of FBS is represented as $\Phi_{\mathrm{FBS}}=\left\{Y_{i}\right\}$. In this paper, the
OFDMA-based LTE system is used and no power control is applied in downlink. Thus, MBS transmission power is depicted by $P_{m}$ and FBS transmission power is depicted by $P_{f}$. To guarantee the quality of signal in heterogeneous networks in terms of the outage probability $\varepsilon$, the signalto-interference-plus-noise ratio (SINR) of MUE and femtocell user (FUE) located at cell edge should be greater than the target SINR $\Gamma_{\text {target. }}$. The amount of resource block (RB) is $N$ where MBS occupies $N_{m}$ and the number of RB that FBS occupied depends on different frequency allocation schemes used by FBS. For example, in orthogonal frequency allocation scheme, $N_{f}=N-N_{m}$. When in the co-channel frequency allocation scheme, $N_{f}=N_{m}=N$. $\rho=N_{m} / N$ denotes the ratio of RB resource that MBS occupies to the overall resources. $\rho=1$ denotes the cochannel frequency allocation scheme is used, while $0<$ $\rho<1$ is the orthogonal frequency allocation scheme. Considering the backhaul among MBS and small cells is non-ideal, the information exchange among them faces the challenge of time delay. Therefore, this paper proposes the cognitive pilot channel (CPC) technique [22] to realize the effective information delivery among MBS and small cells.

\section{Capacity analysis with different frequency allocation schemes}

In this part, three frequency allocation schemes are proposed and downlink capacity of hierarchical network is analyzed in terms of different densities of FBS. A novel dynamic hybrid frequency allocation scheme is proposed to minimize the interference from FBS to MBS.

\subsection{Orthogonal frequency allocation scheme}

In the orthogonal frequency allocation scheme, where different frequency bands are separately assigned to the macrocell and small cell layers. It can decrease the crosslayer interference. As show in Fig. 2, MUEs located at the edge of $\mathrm{MBS}_{0}$ using the specific $\mathrm{RB}$ resource in downlink will receive strong interference from neighbor $\mathrm{MBS}_{i}$. Given the whole number of RB $N$ and available RB $N_{m}=\rho N$, the probability of co-channel interference 


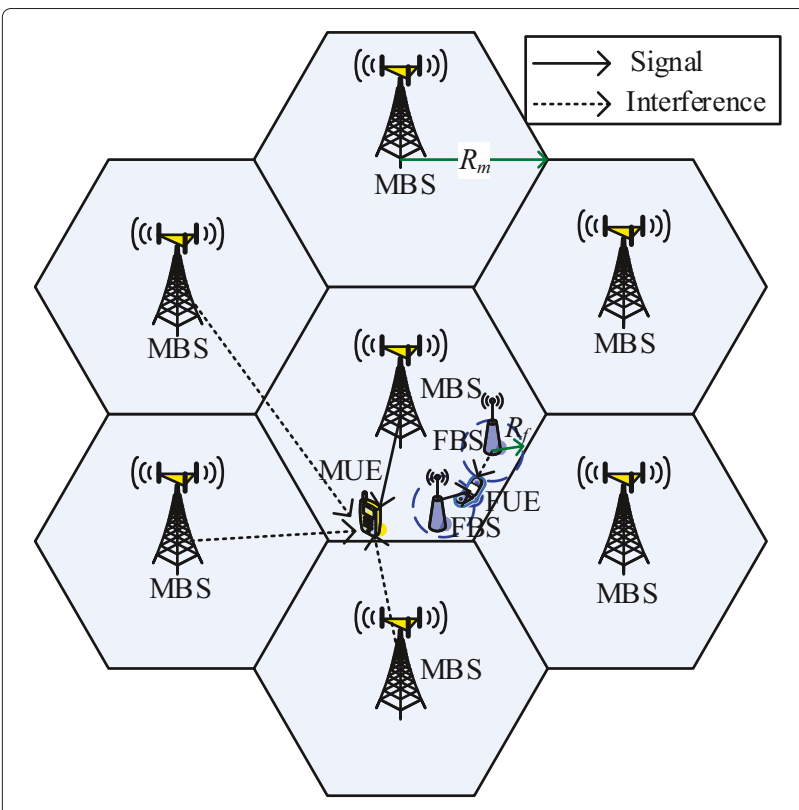

Fig. 2 Scenario of orthogonal frequency allocation scheme

$$
N_{m}=\rho N \geq N_{m, \min }=\frac{\Gamma_{\text {target }}(1-\varepsilon)}{\exp \left(-\frac{\Gamma_{\text {target }} N_{0}}{P_{m} R_{m}^{-\alpha}}\right)-(1-\varepsilon)}
$$

To guarantee the $\mathrm{SINR}_{m}^{\text {orth }}$ quality of MUE with the interrupt probability $\varepsilon$ constraints, the number of $\mathrm{RB}$ should be higher than $N_{m \text {,min }}$. When MBS transmit power $P_{m}=20 \mathrm{~W}$, background noise power $N_{0}=10^{-12} \mathrm{~W}$, $\Gamma_{\text {target }}=3, \varepsilon=0.1$, path loss $\alpha=4$, system bandwidth is $20 \mathrm{MHz}, \mathrm{RB}$ resource $N=110$. Fig. 3 shows how the probability of SINR ${ }_{m}^{\text {orth }}>\Gamma_{\text {target }}$ changes with $\rho$ and $R_{m}$. There is a minimum number of RB resource to guarantee the cell edge MUE's quality. And the minimum number $\mathrm{RB}$ increases when $R_{m}$ increases. When $R_{m}=288 \mathrm{~m}$, $\rho=0.25$, the minimum $N_{m}=28$. When $R_{m}=500 \mathrm{~m}$, $\rho=0.28$, the minimum $N_{m}=31$. When $R_{m}=800 \mathrm{~m}$, $\rho=0.61$, the minimum $N_{m}=68$.

When each MUE occupies one RB resource, the maximum downlink capacity is given by (5).

$$
C_{m}^{\text {orth }}=\log _{2}\left(1+\Gamma_{\text {target }}\right)
$$

2) FUE downlink SINR is depicted by $\operatorname{SINR}_{f}^{\text {orth }}\left(d_{f}\right)$ in (6).

$$
\begin{aligned}
& \operatorname{SINR}_{f}^{\text {orth }}\left(d_{f}\right)=\frac{G_{f} P_{f} d_{f}^{-\alpha}}{N_{0}+\frac{1}{(1-\rho) N} I_{f, f}} \\
& I_{f, f}=\sum_{Y_{i} \in \Phi_{\mathrm{FBS}}^{P} \backslash\left\{Y_{0}\right\}} G_{Y_{i}} P_{f}\left\|Y_{i}\right\|^{-\alpha}
\end{aligned}
$$

$G_{f}$ is the channel gain which is modeled as a negative exponential distribution with mean $1 . d_{f}$ is the distance between FBS and FUE. $I_{f, f}$ denotes the co-channel interference from neighbor FBSs. $G_{Y_{i}}$ and $\left\|Y_{i}\right\|$ depict the channel gain and distance between FBS at location $Y_{i}$ and FUE, respectively, based on the results in $[24,25,27]$ as depicted in (8).

$$
\sum_{I=1}^{6} I_{m_{i}, m}=\sum_{i=1}^{6} G_{m_{i}} P_{m} \bar{d}_{m_{i}, m}^{-\alpha}
$$

$G_{m}$ is the channel gain which is modeled as a negative exponential distribution with mean $1 . P_{m}$ is the transmit power of MBS, $\alpha$ is the pass loss ratio, $N_{0}$ represents the background noise power per RB, $d_{m}$ is the distance between $\mathrm{MBS}_{0}$ and MUE, $I_{m_{i}, m}$ is the co-channel interference to MUE in $\mathrm{MBS}_{0}$ from $\mathrm{MBS}_{i}$, and $\bar{d}_{m_{i}, m}$ is the mean distance between neighboring $\mathrm{MBS}_{i}$ and MUE in $\mathrm{MBS}_{0}$.

To simplify the analysis, it is assumed that only the strongest interference from $\mathrm{MBS}_{k}$ to MUE is considered, where $\bar{d}_{m_{i}, m}=R_{m}$. Then, the coverage radius $R_{m}$ of MBS satisfies (3) in terms of the maximum outage probability of $\varepsilon$.

$$
\begin{aligned}
& P\left(\operatorname{SINR}_{m}^{\text {orth }}\left(R_{m}\right) \geq \Gamma_{\text {target }}\right)=\exp \left(-\frac{\Gamma_{\text {target }} N_{0}}{P_{m} R_{m}^{-\alpha}}\right) \\
& \times \frac{1}{1+\Gamma_{\text {target }} /(\rho N)} \geq 1-\varepsilon
\end{aligned}
$$

$$
\begin{aligned}
E\left[\exp \left(-s I_{f, f}\right)\right] & =\exp \left[-2 \pi \widehat{\lambda}_{\mathrm{FBS}} \int_{0}^{\infty} \frac{u}{1+u^{\alpha} /\left(s P_{f}\right)} d u\right] \\
& =\exp \left(\widehat{\lambda}_{\mathrm{FBS}} p_{f}^{2 / \alpha} s^{2 / \alpha} K_{\alpha}\right)
\end{aligned}
$$

where $K_{\alpha}=2 \pi /[\alpha \sin (2 \pi / \alpha)], \widehat{\lambda}_{\mathrm{FBS}}$ is the maximum density of FBS.

Considering the worst case of the interference from MBS, the SINR of FUE at the cell edge of FBS should be greater than the target SINR threshold $\Gamma_{\text {target }}$ with the outage probability $\varepsilon$ in (9).

$$
P\left(\operatorname{SINR}_{f}^{\text {orth }}\left(R_{f}\right) \geq \Gamma_{\text {target }}\right)=1-\varepsilon
$$

In orthogonal frequency allocation scheme, the maximum density of FBS $\widehat{\lambda}_{\text {FBS }}$ is depicted in (10).

$$
\widehat{\lambda}_{\mathrm{FBS}}=\frac{-\ln (1-\varepsilon)-\frac{\Gamma_{\text {target }}}{P_{f} R_{f}^{-\alpha}} N_{0}}{R_{f}^{2} \Gamma_{\text {target }}^{2 / \alpha} K_{\alpha}(1-\rho)^{-2 / \alpha} N^{-2 / \alpha}}
$$




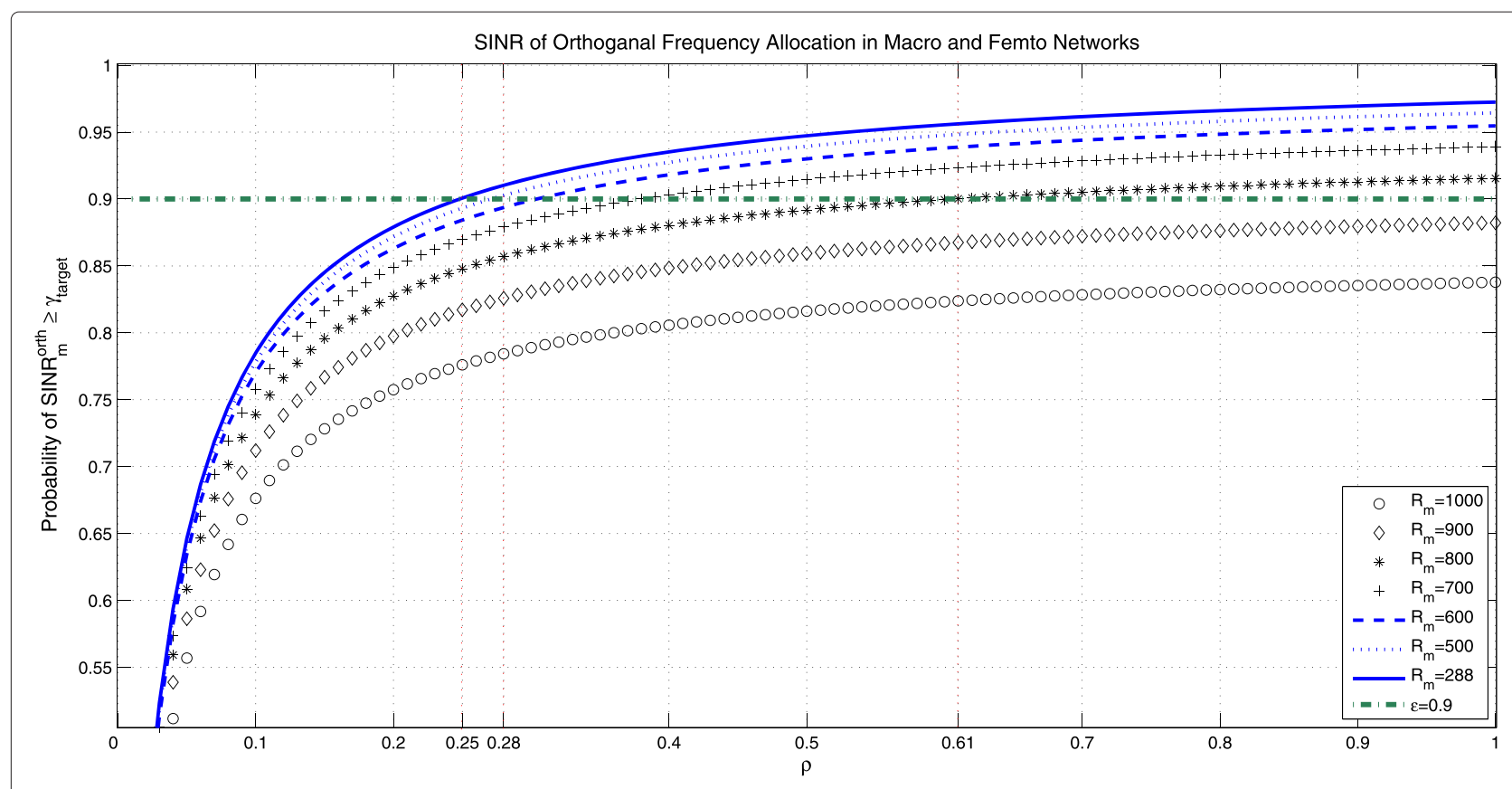

Fig. 3 SINR of MUE for different $\rho$ and $R_{m}$

Thus, the total system capacity is depicted by $C^{\text {orth }}$ in (11).

$$
\begin{aligned}
C^{\text {orth }} & =N_{m} C_{m}^{\text {orth }}+N_{s} C_{s}^{\text {orth }}=N\left[\rho C_{m}^{\text {orth }}+(1-\rho) C_{s}^{\text {orth }}\right] \\
& =N\left[\rho+(1-\rho) \widehat{\lambda}_{\text {FBS }}|H|\right] \log _{2}\left(1+\Gamma_{\text {target }}\right)
\end{aligned}
$$

The maximum density of FBS $\widehat{\lambda}_{\mathrm{FBS}}$ is constrained by the resource ratio $\rho$ and the coverage radius of FBS $R_{f}$, as shown in Fig. 4 . With a fixed $R_{f}$, available RB resources of FBS will decrease with the increase of $\rho$, where interference among different FBS will increase. In order to guarantee the quality of FUE, FBS density should decrease to mitigate the interference by utilizing the spatial isolation among FBSs. Moreover, considering the quality of MUE at the cell edge, FBS density achieves the maximum $\widehat{\lambda}_{\text {FBS, max }}$, when MBS occupies RB resources with the minimum value $\rho_{m, \text { min }}=N_{m, \text { min }} / N$ and FBS has the maximum value $N_{f, \max }=\left(1-\rho_{m, \min }\right) N$. For example, when $R_{m}=$

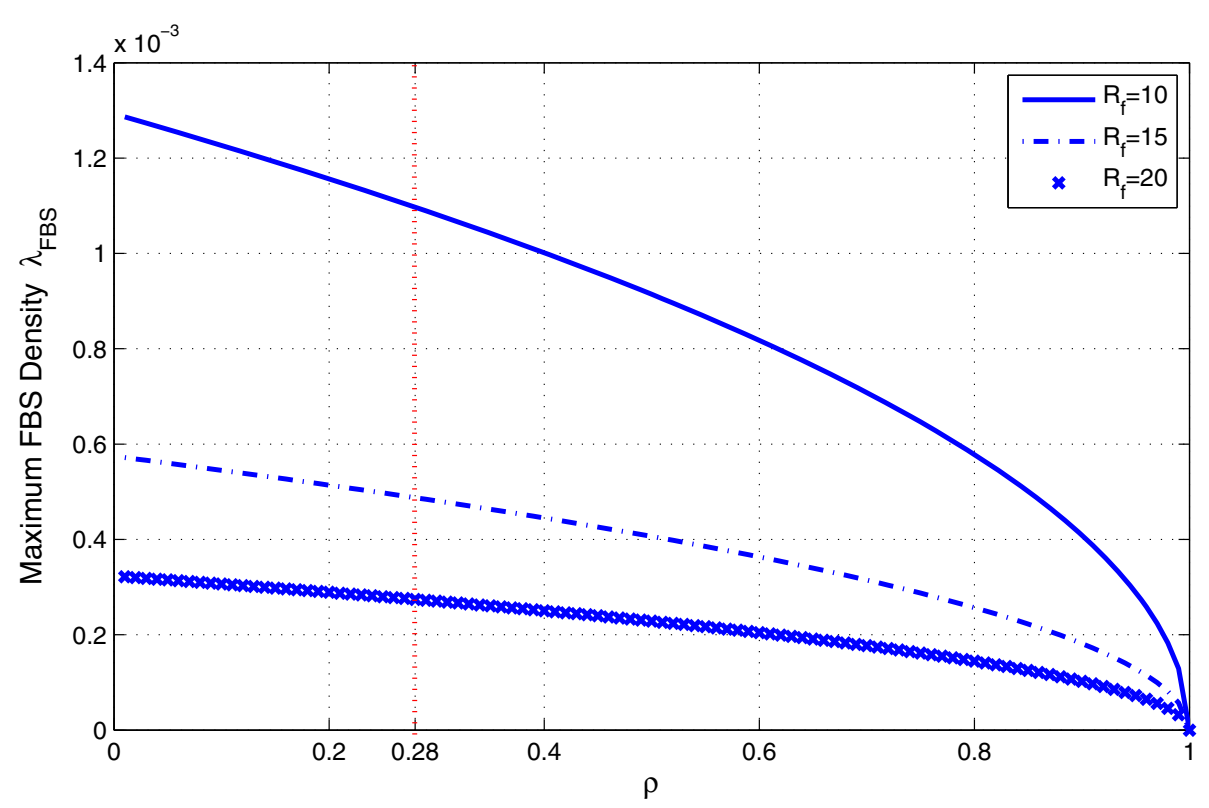

Fig. 4 FBS density $\widehat{\lambda_{\text {FBS }}}$ with different $\rho$ and $R_{f}$ 
$500 \mathrm{~m}, \rho_{m, \min }=0.28$, the maximum $N_{f, \max }=79$. When $R_{f}=10 \mathrm{~m}$, FBS maximum density achieves $\widehat{\lambda}_{\mathrm{FBS}, \max }=$ $1.10 \times 10^{-3}$. When $R_{f}=20 \mathrm{~m}$, FBS maximum density achieves $\widehat{\lambda}_{\text {FBS, max }}=2.74 .10 \times 10^{-4}$. Thus, both RB resource ratio $\rho$ and coverage radius $R_{f}$ will affect the maximum FBS density in orthogonal frequency allocation scenario.

When each FBS occupies one RB resource, the downlink capacity is given by (12).

$$
C_{f}^{\text {orth }}=\widehat{\lambda}_{\text {FBS }}|H| \log _{2}\left(1+\Gamma_{\text {target }}\right)
$$

Therefore, when system bandwidth is $N, \mathrm{RB}$ resource ratio is $\rho=N_{m} / N$, the total downlink capacity is given by (13).

$$
\begin{aligned}
C^{\text {orth }} & =N_{m} C_{m}^{\text {orth }}+N_{f} C_{f}^{\text {orth }} \\
& =N\left[\rho+(1-\rho) \widehat{\lambda}_{\mathrm{FBS}}|H|\right] \log _{2}\left(1+\Gamma_{\text {target }}\right)
\end{aligned}
$$

Furthermore, the maximum total downlink capacity is defined as an optimization problem in (14), when the optimal $\rho$ is chosen.

$$
\begin{gathered}
\rho^{*}=\operatorname{argmax}\left(\mathrm{C}^{\text {orth }}\right) \\
\text { s.t. } N_{m, \min } / N \leq \rho<1 \\
\because C^{\text {orth }}=A_{1}\left[\rho+B_{1}(1-\rho)^{1+2 / \alpha}\right] \\
\therefore d C^{\text {orth } / d \rho=}=A_{1}\left[1-B_{1}(1+2 / \alpha)(1-\rho)^{2 / \alpha}\right]=0 \\
\therefore \rho_{\min }=1-\left[\frac{1}{B_{1}(1+2 / \alpha)}\right]^{\alpha / 2}
\end{gathered}
$$

where $B_{1}=|H| N^{2 / \alpha}\left[-\ln (1-\varepsilon)-\Gamma_{\text {target }} N_{0} / P_{f} R_{f}^{-\alpha}\right] /$ $\left(R_{f}^{2} \Gamma_{\text {target }}^{2 / \alpha} / K_{\alpha}\right)$ and $A_{1}=N \log _{2}\left(1+\Gamma_{\text {target }}\right)=2 N$. Therefore, the maximum value $C^{\text {orth }}$ is achieved at $\rho=$ $N_{m, \min } / N$ as shown in Fig. 5.

To ensure the quality of MUE and FUE, the total downlink capacity $C^{\text {orth }}$ achieves the maximum $C^{\text {orth }}=1.13 \times$ $10^{5} \mathrm{bit} / \mathrm{s}$ when FBS density is $\widehat{\lambda}_{\mathrm{FBS} \text {, max }}=1.10 \times 10^{-3}$, where $R_{m}=500 \mathrm{~m}, \rho \geq \rho_{m, \min }=0.28$, and $R_{f}=$ $10 \mathrm{~m}$. When $R_{f}=20 \mathrm{~m}$, the total downlink capacity $C^{\text {orth }}=2.83 \times 10^{4} \mathrm{bit} / \mathrm{s}$ when FBS density is $\widehat{\lambda}_{\mathrm{FBS} \text {, max }}=$ $2.74 \times 10^{-4}$.

\subsection{Co-channel frequency allocation scheme}

Although orthogonal frequency allocation scheme can suppress the co-channel interference among macrocells and small cells, it reduces the spectral efficiency. In terms of the scarce spectrum resources, co-channel frequency allocation scheme has been paid much attention recently to improve the spectral efficiency. In terms of densely deployed small cells, new types of interference are brought forward, such as the interference from FBS to nearby MUE in downlink and neighboring MBS to MUE at the cell edge. Moreover, FUE at the cell edge will suffer from the strong co-channel interference from neighboring MBS in downlink. Therefore, the density of FBS is a key factor that will affect the capacity of hierarchical networks using the co-channel frequency allocation scheme.

Therefore, this section will analyze key influential factors to FBS density and find out the optimal FBS density to

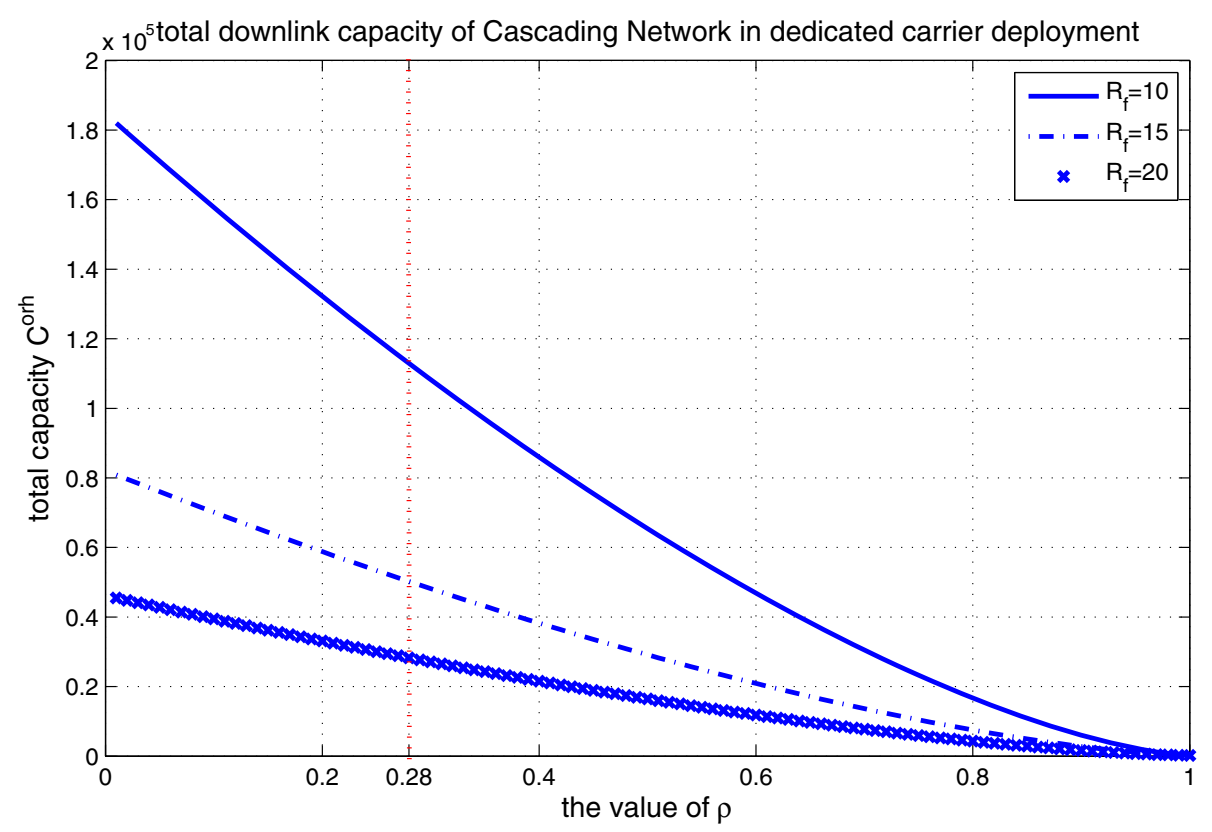

Fig. 5 System capacity in orthogonal frequency allocation scheme with different $\rho$ and $R_{m}$ 
improve the downlink capacity of hierarchical networks. Different types of interference are depicted in Fig. 6. In terms of RB resources of $N$, the probability of MUE that suffers co-channel interference from $\mathrm{MBS}_{i}$ is $1 / N$. The probability of FUE that sufferers co-channel interference from neighboring FBSs is $1 / N$.

1) MUE downlink SINR is depicted by $\operatorname{SINR}_{m}^{\text {coch }}\left(d_{m}\right)$ in (16).

$$
\begin{aligned}
\operatorname{SINR}_{m}^{\operatorname{coch}}\left(d_{m}\right) & =\frac{G_{m} P_{m} d_{m}^{-\alpha}}{N_{0}+\frac{1}{N} \sum_{i=1}^{6} I_{m_{i}, m}+\frac{1}{N} I_{f, m}} \\
\sum_{i=1}^{6} I_{m_{i}, m} & =\sum_{i=1}^{6} G_{m_{i}} P_{m} \bar{d}_{m_{i}, m}^{-\alpha} \\
I_{f, m} & =\sum_{Y_{i} \in \Phi_{\mathrm{FBS}}^{P}} G_{Y_{i}} P_{s}\left\|Y_{i}\right\|^{-\alpha}
\end{aligned}
$$

$I_{f, m}$ is the co-channel interference from FBS to MUE in $\mathrm{MBS}_{0} . I_{m_{i}, m}$ is the co-channel interference from neighboring $\mathrm{MBS}_{i}$ to MUE in $\mathrm{MBS}_{0} . \bar{d}_{m_{i}, m}$ depicts the mean distance for MUEs in $\mathrm{MBS}_{i}$ and $\mathrm{MBS}_{0}$.

Considering the SINR of MUE at cell edge with the outage probability constraint $\varepsilon, \operatorname{SINR}_{m}^{\text {coch }}$ satisfies the (18).

$$
P\left(\operatorname{SINR}_{m}^{\text {coch }}\left(R_{f}\right) \geq \Gamma_{\text {target }}\right)=1-\varepsilon
$$

By applying (16) in to (18), the maximum density of FBS $\widehat{\lambda}_{\mathrm{FBS}, \mathrm{MUE}}$ is depicted in (19).

$$
\tilde{\lambda}_{\mathrm{FBS}, \mathrm{MUE}}=\frac{-\ln \left[(1-\varepsilon)\left(1+\frac{\Gamma_{\text {target }}}{N}\right)\right]-\frac{\Gamma_{\text {target }} N_{0}}{P_{m} R_{m}^{-\alpha}}}{N^{-\frac{2}{\alpha}} R_{m}^{2}\left(\frac{P_{f} \Gamma_{\text {target }}}{P_{m}}\right)^{2 / \alpha} K_{\alpha}}
$$

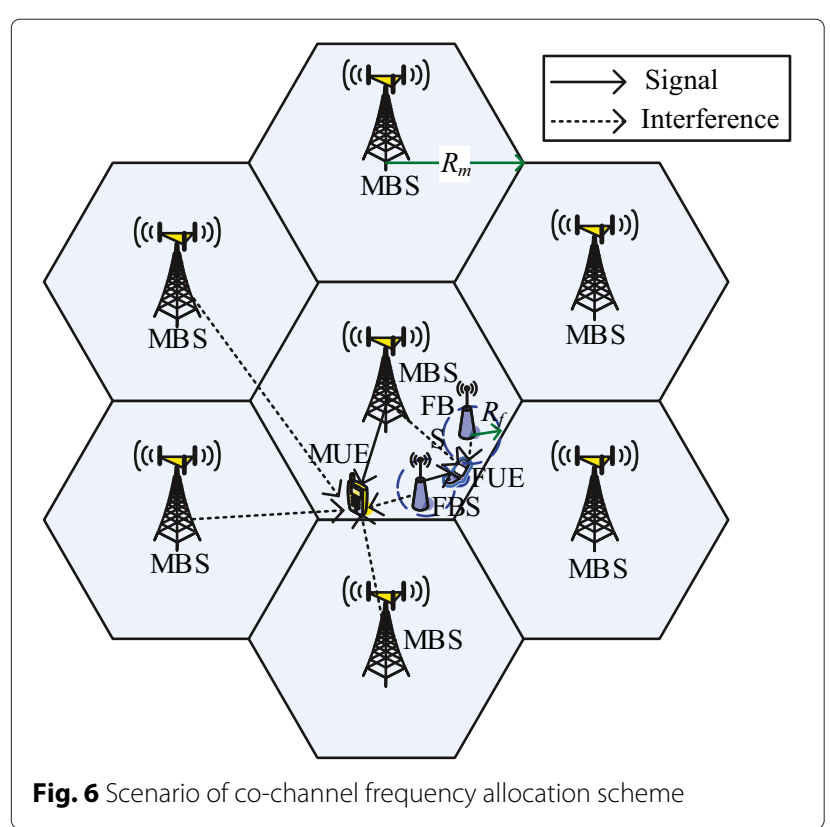

2) Similarly, SINR of FUE at cell edge with the outage probability constraint $\varepsilon$ is depicted by $\operatorname{SINR}_{f}^{\text {coch }}\left(d_{f}\right)$ in (20).

$$
\begin{aligned}
& \operatorname{SINR}_{f}^{\mathrm{coch}}\left(d_{f}\right)=\frac{G_{f} P_{f} d_{f}^{-\alpha}}{N_{0}+\frac{1}{N} I_{f, f}+\frac{1}{N} \sum_{i=0}^{6} I_{m_{i}, f}} \\
& I_{f, f}=\sum_{Y_{i} \in \Phi_{\mathrm{FBS}}^{p} \backslash\left\{Y_{0}\right\}} G_{Y_{i}} P_{f} Y_{i}^{-\alpha} \\
& \sum_{i=0}^{6} I_{m_{i}, f}=\sum_{i=0}^{6} G_{m_{i}} P_{m} \bar{d}_{m_{i}, f}^{-\alpha}
\end{aligned}
$$

$I_{f, f}$ is the co-channel interference from neighboring FBS in the same MBS. $I_{m_{i}, f}$ is the co-channel interference from neighboring $\mathrm{MBS}_{i} \cdot \bar{d}_{m_{i}, f}$ depicts the mean distance for FUEs in $\mathrm{MBS}_{i}$ and $\mathrm{MBS}_{0}$.

Considering the SINR of FUE at cell edge with the outage probability constraint $\varepsilon$, $\operatorname{SINR}_{f}^{\text {coch }}$ satisfies (23).

$$
P\left(\operatorname{SINR}_{f}^{\text {coch }}\left(R_{f}\right) \geq \Gamma_{\text {target }}\right)=1-\varepsilon
$$

The maximum density of FBS $\widehat{\lambda}_{\mathrm{FBS}, \mathrm{FUE}}$ is depicted in (24).

$\bar{\lambda}_{\mathrm{FBS}, \mathrm{FUE}}=\frac{-\ln \left[(1-\varepsilon)\left(1+\frac{\Gamma_{\text {target }} P_{m} R_{m}^{-\alpha}}{P_{f} R_{f}^{-\alpha} N}\right)^{2}\right]-\frac{\Gamma_{\text {target }} N_{0}}{P_{f} R_{f}^{-\alpha}}}{N^{-\frac{2}{\alpha}} R_{f}^{2} \Gamma_{\text {target }^{2 / \alpha} K_{\alpha}}}$

Therefore, $\bar{\lambda}_{\mathrm{FBS}}=\min \left(\bar{\lambda}_{\mathrm{FBS}, \mathrm{MUE}}, \bar{\lambda}_{\mathrm{FBS}, \mathrm{FUE}}\right)$ depicts the maximum density of FBS. In terms of system bandwidth of $N$, FBS density is $\bar{\lambda}_{\mathrm{FB}}$, the achievable downlink capacity is depicted in (25).

$$
C^{\text {coch }}=N\left(1+\bar{\lambda}_{\mathrm{FBS}}|H|\right) \log _{2}\left(1+\Gamma_{\text {target }}\right)
$$

Therefore, two key factors restricting downlink capacity for co-channel frequency allocation are RB resources and the density of FBS. The downlink capacity increases with the increase of RB as depicted in Fig. 7. And a maximum density exists which is mainly determined by MUE's tolerance to co-channel interference.

\subsection{Hybrid frequency allocation scheme}

Based on the analysis of orthogonal and co-channel frequency allocation schemes, the FBS density $\lambda_{\text {FBS }}$ and optimal RB resource ratio $\rho$ will affect the capacity of hierarchical networks. However, the self-deployment and uncertainty of switch-on and switch-off features of FBS in practice are key challenges. Besides the time delay of information, exchange among hierarchical networks via a third party backhaul is another problem. Therefore, it is difficult to make an optimal resource allocation scheme based on the FBS density in a practical scenario. Moreover, the co-channel frequency allocation scheme can provide an 


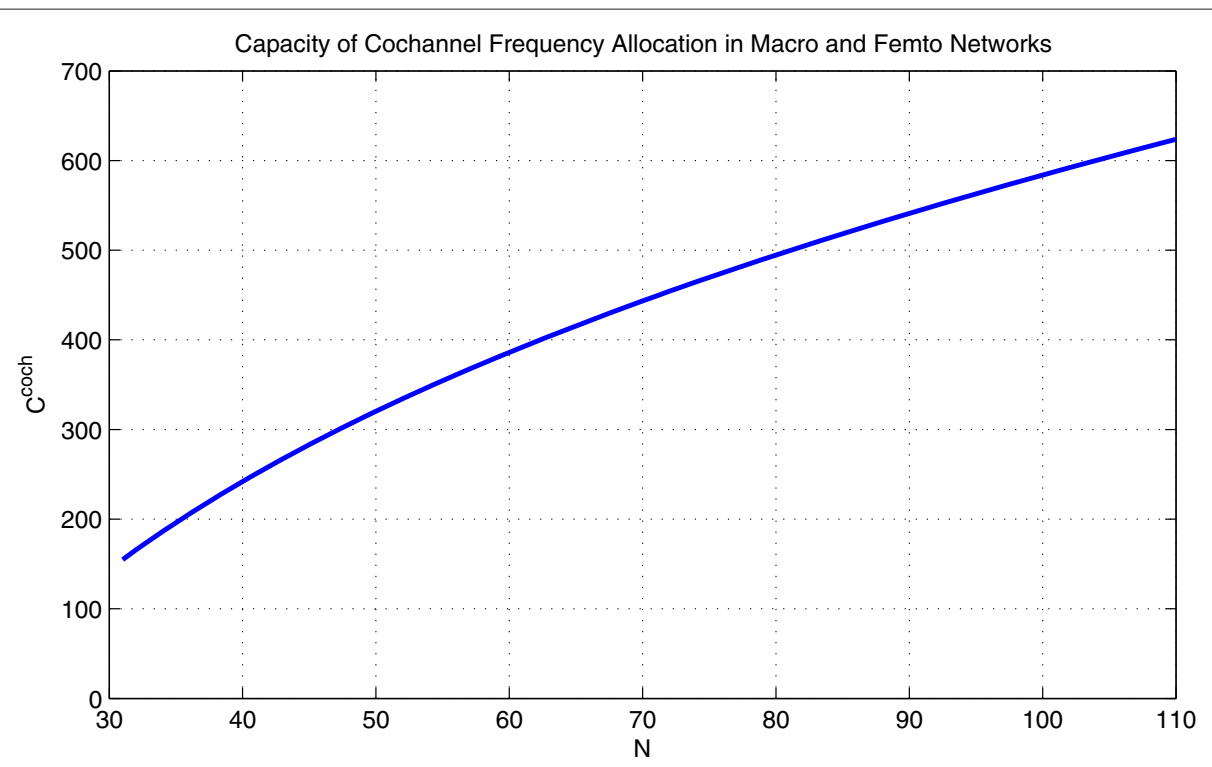

Fig. 7 System capacity in co-channel deployment for different $N$

efficient spectrum utilization solution, but it inevitably introduces the strong co-channel interference which will decrease the system capacity.

Therefore, a dynamic hybrid frequency allocation scheme is proposed to increase the downlink capacity, by taking into account the advantages of both orthogonal and co-channel frequency allocation schemes. The coverage of MBS is divided into inner and outer circle regions as shown in Fig. 8. In the inner circle region, the FBS applies the orthogonal frequency allocation scheme. In the outer

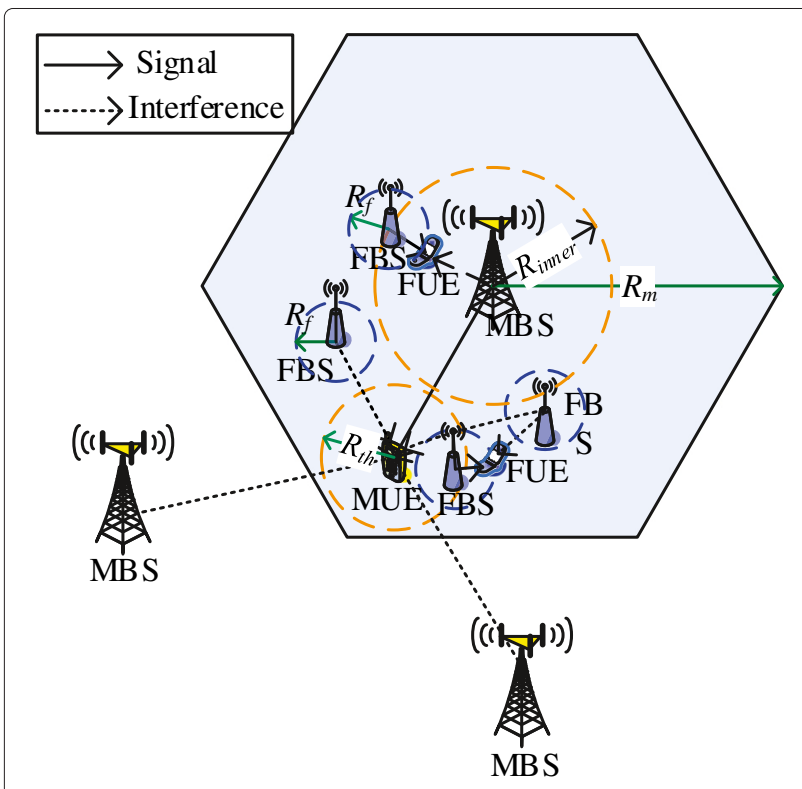

Fig. 8 Scenario of hybrid frequency allocation scheme circle region, the FBS applies the orthogonal frequency allocation scheme when it is within a threshold radius $R_{\text {th }}$ of MUE and applies the co-channel frequency allocation scheme when it is outside a threshold radius $R_{\text {th }}$ of MUE. Thus, the hybrid frequency allocation scheme is described and analyzed in detail below, which can improve the downlink capacity by increasing the density of FBS and improving the frequency reuse.

1) Capacity analysis of the inner circle region

To simplify the theoretical analysis and mathematical calculations, it is assumed that FUE will suffer from the strong co-channel interference from the serving $\mathrm{MBS}_{0}$ and the interference from neighboring $\operatorname{MBS}_{k}(k=$ $1,2, \ldots, 6)$ is ignored. The SINR of FUE is denoted by $\operatorname{SINR}_{f \text {,inner }}^{\text {hybrid }}$ in (26).

$$
\operatorname{SINR}_{f, \text { inner }}^{\text {hybrid }}\left(d_{f}\right)=\frac{G_{f} P_{f} d_{f}^{-\alpha}}{N_{0}+G_{m} P_{m} d_{m}^{-\alpha}}
$$

where $d_{f}$ represents the distance between FBS and FUE and $d_{m}$ represents the distance between MBS and FUE. To simplify the analysis, the effect of channel gain from $G_{f}$ and $G_{m}$ to $\operatorname{SINR}_{f \text {,inner }}^{\text {hybrid is ignored. When FUE is located }}$ on the edge of FBS as depicted by $d_{f}=R_{f}$, $\operatorname{SINR}_{f \text {,inner }}^{\text {hybrid }}$ is denoted in (27).

$$
\operatorname{SINR}_{f, \text { inner }}^{\text {hybrid }}\left(R_{f}\right)=\frac{P_{f} R_{f}^{-\alpha}}{N_{0}+P_{m} d_{m}^{-\alpha}}=\Gamma_{\text {target }}
$$

When $\alpha=4$ and $P_{f} R_{f}^{-\alpha}-N_{0} \Gamma_{\text {target }}>0$, the radius of FBS $R_{f}$ should satisfy $R_{f}<\left(P_{f} / N_{0} \Gamma_{\text {target }}\right)^{1 / 4}=4.27 \times 10^{2}$ 
m. The radius $R_{\text {inner }}$ of the inner cell region is depicted in (28).

$$
R_{\text {inner }}=d_{m}+R_{f}=R_{f}+\left(\frac{\Gamma_{\text {target }} P_{m}}{P_{f} R_{f}^{-\alpha}-N_{0} \Gamma_{\text {target }}}\right)^{1 / \alpha}
$$

When $R_{f}$ is small and the effect of the background noise to $R_{\text {inner }}$ can be ignored, the $R_{\text {inner }}$ is simplified as $R_{\text {inner }} \leq$ $R_{f}\left[1+\left(\Gamma_{\text {target }} P_{m} / P_{f}\right)^{1 / 4}\right]=5.95 R_{f} \leq R_{m}$. Furthermore, the total number of RB is $N$ and $(1-\rho) N$ is assigned to FBS. Considering the SINR of MUE at the cell edge $\left(d_{m}=\right.$ $R_{\text {inner }}$ ) with the outage probability constraint $\varepsilon$, the $R_{\text {inner }}$ is depicted in (29)-(30).

$P\left(\operatorname{SINR}_{m}^{\text {hybrid }}\left(R_{\text {inner }}\right)=G_{m} P_{m} R_{\text {inner }}^{-\alpha} / N_{0} \geq \Gamma_{\text {target }}\right) \geq 1-\varepsilon$

$$
R_{\text {inner }} \leq\left[-\ln (1-\varepsilon) P_{m} /\left(N_{0} \Gamma_{\text {target }}\right)\right]^{1 / \alpha}
$$

Similarly, considering the SINR of FUE at the cell edge $\left(d_{m}=R_{\text {inner }}\right)$ with the outage probability constraint $\varepsilon$, the SINR of FUE is depicted in (31).

$$
P\left(\operatorname{SINR}_{f}^{\text {hybrid }}\left(R_{f}\right)=\frac{G_{f} P_{f} R_{f}^{-\alpha}}{N_{0}+I_{f, f}^{\text {hybrid }} /(1-\rho) N} \geq \Gamma_{\text {target }}\right)=1-\varepsilon
$$

$$
\begin{gathered}
I_{f, f}^{\text {hybrid }}=\sum_{Y_{i} \in \Phi_{\mathrm{FBS}}^{p} \backslash\left\{Y_{0}\right\}, Y_{i} \leq 2 R_{\text {inner }}} G_{Y_{i}} P_{f}\left\|Y_{i}\right\|^{-\alpha} \\
E\left[\exp \left(-\mathrm{sI}_{\mathrm{f}, \mathrm{f}}^{\text {hybrid }}\right)\right]= \\
\exp \left[-\pi \bar{\lambda}_{\mathrm{FBS}}^{\mathrm{R}_{\text {inner }}}\left(\mathrm{sP}_{\mathrm{f}}\right)^{1 / 2} \tan ^{-1}\right. \\
\left.\left(4 R_{\text {inner }}^{2} /\left(s P_{f}\right)^{1 / 2}\right)\right]
\end{gathered}
$$

where $s=\Gamma_{\text {target }} /\left[P_{f} R_{f}^{-\alpha}(1-\rho) N\right]$. By putting (32) and (33) into (31), the SINR of FUE is denoted in (35).

$$
\begin{aligned}
& P\left(\operatorname{SINR}_{f}^{\text {hybrid }}\left(R_{f}\right) \geq \Gamma_{\text {target }}\right) \\
& =\exp \left[-\mathrm{N}_{0} \Gamma_{\text {target }} /\left(\mathrm{P}_{\mathrm{f}} \mathrm{R}_{\mathrm{f}}^{-\alpha}\right)\right] \\
& \exp \left[-\pi \bar{\lambda}_{\text {FBS }}^{- \text {Rinner }}\left(\mathrm{sP}_{\mathrm{f}}\right)^{1 / 2} \tan ^{-1}\left(4 \mathrm{R}_{\text {inner }}^{2} /\left(\mathrm{sP}_{\mathrm{f}}\right)^{1 / 2}\right)\right]=1-\varepsilon
\end{aligned}
$$

Therefore, the density of FBS in the inner circle region is denoted by $\bar{\lambda}_{\mathrm{FBS}}^{R_{\text {inner }}}$ in (35).

$$
\bar{\lambda}_{\mathrm{FBS}}^{R_{\text {inner }}}=\frac{-\ln (1-\varepsilon)-N_{0} \Gamma_{\text {target }} /\left(P_{f} R_{f}^{-\alpha}\right)}{\pi \sqrt{\Gamma_{\text {target }} R_{f}^{\alpha} /[(1-\rho) N]} \tan ^{-1}\left\{4 R_{\text {inner }}^{2} / \sqrt{\Gamma_{\text {target }} R_{f}^{\alpha} /[(1-\rho) N]}\right\}}
$$

The downlink capacity of inner circle region of hybrid frequency allocation scheme is depicted in (36).

$$
C_{\text {inner }}^{\text {hybrid }}=N\left[\rho+(1-\rho) \bar{\lambda}_{\text {FBS }}^{R_{\text {inner }}}\left|H_{\text {inner }}\right|\right] \log _{2}\left(1+\Gamma_{\text {target }}\right)
$$

where $\left|H_{\text {inner }}\right|=\pi R_{\text {inner }}^{2}$. When $N=110, \rho=0.28$, $\bar{\lambda}_{\mathrm{FBS}}^{R_{\text {inner }}}=1.10 \times 10^{-3}, R_{\text {inner }}=59.5 \mathrm{~m}$, the downlink capacity is $C_{\text {inner }}^{\text {hybrid }}=2.0 \times 10^{3} \mathrm{bit} / \mathrm{s}$.

2) Capacity analysis of the outer circle region

In the outer circle region of MBS, MUE will suffer from strong interferences from FBSs in the vicinity. Thus, FBS will apply the restricted co-channel frequency allocation scheme to be aware of the available RB resources in different locations by adding cognitive abilities. FBS will minimize the co-channel interference to MUE and increase the deployment density of small cells. Within a threshold radius $R_{\text {th }}$ of MUE, the orthogonal frequency allocation scheme is applied to FBS to minimize the interference. Otherwise, the co-channel frequency allocation scheme is applied to FBS in the outer circle region of MBS. In terms of the maximum outage probability of $\varepsilon$, the SINR of MUE is depicted by $\operatorname{SINR}_{m}^{\text {hybrid }}\left(d_{m}\right)$ in (37), which includes the interference from neighboring MBS and FBS outside a threshold radius of $R_{\text {th }}$.

$$
\operatorname{SINR}_{m}^{\text {hybrid }}\left(d_{m}\right)=\frac{G_{m} P_{m} d_{m}^{-\alpha}}{N_{0}+\frac{1}{N} \sum_{i=1}^{6} I_{m_{i}, m}+\frac{1}{N} I_{f, m}^{\text {hybrid }}}
$$

$$
\begin{aligned}
& \sum_{i=1}^{6} I_{m_{i}, m}=\sum_{i=1}^{6} G_{m_{i}} P_{m} \bar{d}_{m_{i}, m}^{-\alpha} \\
& I_{f, m}^{\text {hybrid }}=\sum_{Y_{i} \in \Phi_{\mathrm{FBS}}^{p},\left\|Y_{i}\right\| \geq R_{t h}} G_{Y_{i}} P_{f}\left\|Y_{i}\right\|^{-\alpha}
\end{aligned}
$$

where $I_{f, m}^{\text {hybrid }}$ depicts the interference from FBS outside the threshold radius $R_{\text {th }}$ to MUE, $I_{m_{i}, m}$ represents the interference from neighboring $\mathrm{MBS}_{i}$ to MUE in $\mathrm{MBS}_{0}, \bar{d}_{m_{i}, m}$ is the mean distance between $\mathrm{MBS}_{i}$ and MUE in $\mathrm{MBS}_{0}$. When $\alpha=4$, the calculations can be simplified as denoted in (40).

$$
\begin{aligned}
E\left[\exp \left(-\mathrm{sI}_{\mathrm{f}, \mathrm{m}}^{\text {hybrid }}\right)\right]= & \exp \left\{-\pi \bar{\lambda}_{\mathrm{FBS}}^{-\mathrm{R}_{\mathrm{th}}}\left(\mathrm{sP}_{\mathrm{f}}\right)^{1 / 2}\right. \\
& {\left.\left[\pi / 2-\tan ^{-1}\left(\mathrm{R}_{\mathrm{th}}^{2} /\left(\mathrm{sP}_{\mathrm{f}}\right)^{1 / 2}\right)\right]\right\} }
\end{aligned}
$$

In terms of the outage probability constraint $\varepsilon$, the SINR of MUE at the cell edge of MBS is depicted in (41).

$$
P\left(\operatorname{SINR}_{m}^{\text {hybrid }}\left(R_{m}\right) \geq \Gamma_{\text {target }}\right) \geq 1-\varepsilon
$$

By putting (37) and (40) into (41), the SINR of MUE is denoted in (42), where only one strong interference from 
$\mathrm{MBS}_{k}$ is considered and $\bar{d}_{m_{i}, m}=R_{m}$. Furthermore, the density of FBS is denoted by $\bar{\lambda}_{\mathrm{FBS}, \mathrm{MUE}}^{R_{\mathrm{th}}}$ in (43).

$$
\begin{aligned}
& P\left(\operatorname{SINR}_{m}^{\text {hybrid }}\left(R_{m}\right) \geq \Gamma_{\text {target }}\right) \\
& =\frac{\exp \left(-\frac{\Gamma_{\text {target }} N_{0}}{P_{m} R_{m}^{-4}}\right)}{1+\Gamma_{\text {target }} / N} \exp \left\{-\pi \bar{\lambda}_{\mathrm{FBS}, \mathrm{MUE}}^{R_{\text {th }}} R_{m}^{2} \sqrt{\frac{P_{f} \Gamma_{\text {target }}}{N P_{m}}}(42)\right. \\
& \left.\left[\frac{\pi}{2}-\tan ^{-1}\left(R_{\mathrm{th}}^{2} R_{m}^{-2} / \sqrt{\frac{P_{f} \Gamma_{\text {target }}}{N P_{m}}}\right)\right]\right\} \geq 1-\varepsilon \\
& \bar{\lambda}_{\mathrm{FBS}, \mathrm{MUE}}^{R_{\text {th }}}=\frac{-\ln \left[(1-\varepsilon)\left(1+\frac{\Gamma_{\text {target }}}{N}\right)\right]-\frac{\Gamma_{\text {target }} N_{0}}{P_{m} R_{m}^{-4}}}{\pi R_{m}^{2} \sqrt{\frac{P_{f} \Gamma_{\text {target }}}{N P_{m}}}\left[\frac{\pi}{2}-\tan ^{-1}\left(R_{\mathrm{th}}^{2} R_{m}^{-2} / \sqrt{\frac{P_{f} \Gamma_{\text {target }}}{N P_{m}}}\right)\right]}
\end{aligned}
$$

Thus, the downlink capacity in the outer circle region is depicted by $C_{\text {outer }}^{\text {hybrid }}$ in (44).

$$
C_{\text {outer }}^{\text {hybrid }}=N\left(1+\bar{\lambda}_{\text {FBS }}^{\text {outer }}\left|H_{\text {outer }}\right|\right) \log _{2}\left(1+\Gamma_{\text {target }}\right)
$$

where $\left|H_{\text {outer }}\right|=|H|-\left|H_{\text {inner }}\right|=\frac{3 \sqrt{3} R_{m}^{2}}{2}-\pi R_{\text {inner }}^{2}$ represents the outer circle region. And the total downlink capacity of inner and outer circle regions are denoted by $C_{\text {total }}^{\text {hybrid }}$ in (46).

$$
\begin{aligned}
& C_{\text {total }}^{\text {hybrid }}=C_{\text {inner }}^{\text {hybrid }}+C_{\text {outer }}^{\text {hybrid }} \\
= & {\left[\rho+(1-\rho) \bar{\lambda}_{\mathrm{FBS}}^{R_{\text {inner }}}\left|H_{\text {inner }}\right|+1+\bar{\lambda}_{\mathrm{FBS}}^{\text {outer }}\left|H_{\text {outer }}\right|\right] } \\
& N \log _{2}\left(1+\Gamma_{\text {target }}\right)
\end{aligned}
$$

In terms of different RB resources $N$ and the threshold radius $R_{\mathrm{th}}$, the downlink capacity of hybrid frequency allocation scheme and co-channel frequency allocation scheme is compared as depicted in Fig. 9. The downlink capacity will increase with the increase of $R_{\text {th }}$ in the outer circle region. By using the orthogonal frequency allocation scheme for FBS within the threshold radius $R_{\text {th }}$ of MUE in the outer circle region, the strong interference from FBS to MUE in the vicinity can be greatly minimized, which can improve the downlink capacity. Therefore, the proposed hybrid frequency allocation scheme can effectively minimize the cross layer interference in hierarchical networks. When $N=110, \rho=0.28, R_{\text {inner }}=59.5 \mathrm{~m}$, $R_{\mathrm{th}}=50 \mathrm{~m}, \bar{\lambda}_{\mathrm{FBS}}^{R_{\text {inner }}}=1.10 \times 10^{-3}, \bar{\lambda}_{\mathrm{FBS}}^{R_{\text {outer }}}=8.73 \times 10^{-6}$, the theoretical maximum downlink capacity can achieve $C_{\text {total }}^{\text {hybrid }}=3.45 \times 10^{3} \mathrm{bit} / \mathrm{s}$.

In summary, based on the analysis above, there are four key factors that will affect the downlink capacity of hierarchical networks. First, $R_{\text {inner }}$ is used to identify the inner and outer cell regions, which is directly related to FBS coverage radius $R_{f}$. Second, the threshold radius $R_{\text {th }}$ of MUE will affect the downlink capacity which increases with the increase of $R_{\text {th }}$. Third, the RB resource $N$ will affect the network capacity which will increase with the increase of $N$. Fourth, the network capacity will increase with the increase of $\bar{\lambda}_{\text {FBS }}$. Therefore, by optimal parameters design

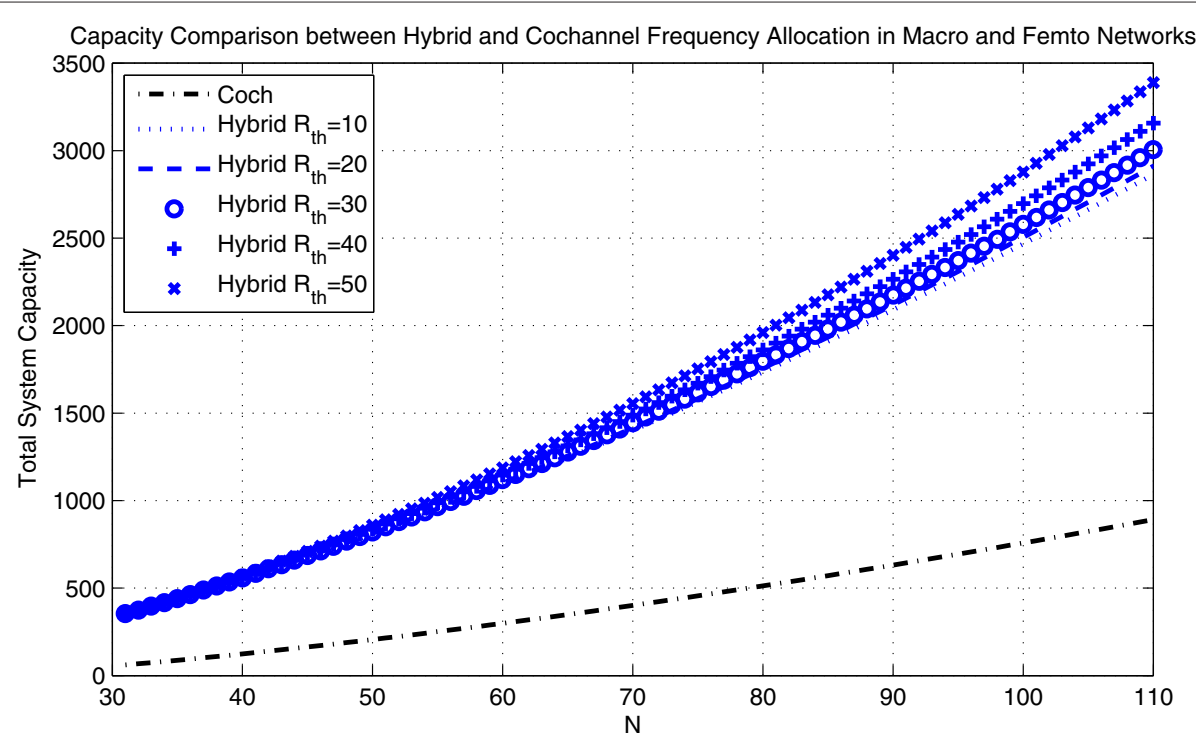

Fig. 9 Total capacity (hybrid vs. co-channel deployment) 
in the hybrid frequency allocation scheme, co-channel interference can be greatly minimized, which can increase the FBS deployment density and improve the network capacity.

\section{Optimal geographic region division scheme}

According to 3GPP TR 36.814 [28], MBS and FBS are defined as different layers and the information exchange among them are limited due to the $\mathrm{X} 2$ interface and backhaul delay. Therefore, this paper has proposed a feasible solution by using CPC technology to support the information exchange among MBS and FBS. By adding the cognitive functions, the proposed solution applies the in-band $\mathrm{CPC}$ technology [22] to deliver the frequency occupancy information of MUE according to different geographic zones. Thereafter, the frequency self-deployment of FBS is carried out to improve the downlink capacity of hierarchical networks.

\subsection{Optimal zones division scheme in different geographic regions}

According to the analysis in Section 3, the FBS deployment density $\bar{\lambda}_{\mathrm{FBS}}$ is one of the key factors that will affect the downlink capacity. By effectively utilizing the small coverage feature of FBS, an optimal FBS deployment can improve the efficiency of frequency reuse to enhance the network capacity.

As shown in Fig. 10a, by dividing the MBS coverage area into uniform square geographic regions, small cells can be aware of the frequency occupancy information of local zones via CPC. Therefore, FBS can utilize orthogonal frequency resources that is different from neighboring MUE to avoid co-channel interference and improve the network capacity. Considering the uncertainty and randomness of small cell coverage areas, the square geographic zones cannot ideally match the contour of network coverage areas, which will lead to errors using different geographic region division schemes. Thus, in this section,
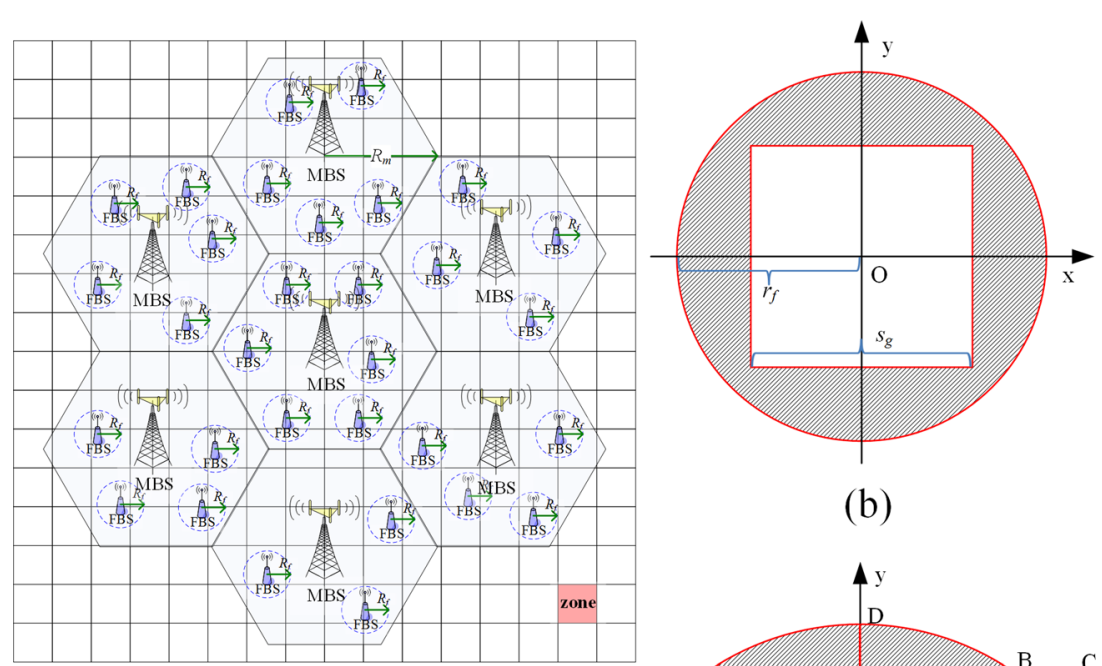

(b)

(a)

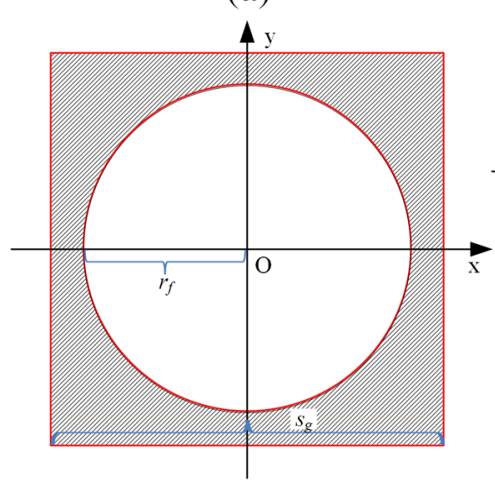

(d)

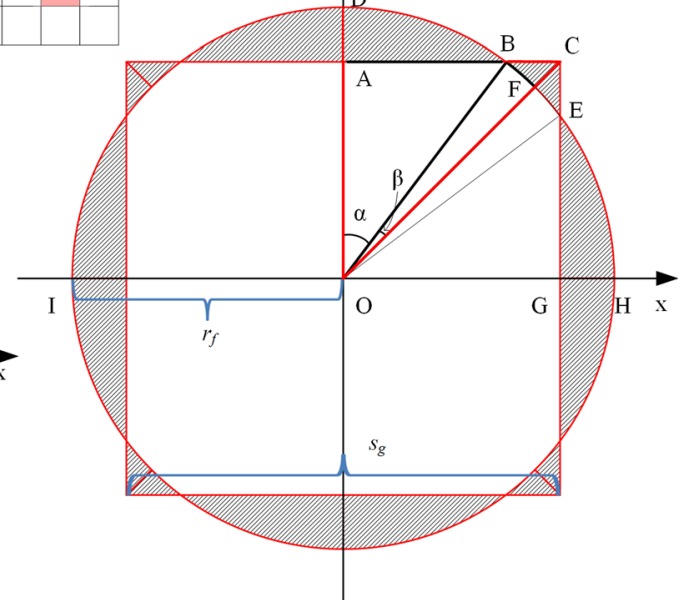

(c)

Fig. 10 Scenario of optimal zone division in different geographical regions (a)-(d) 


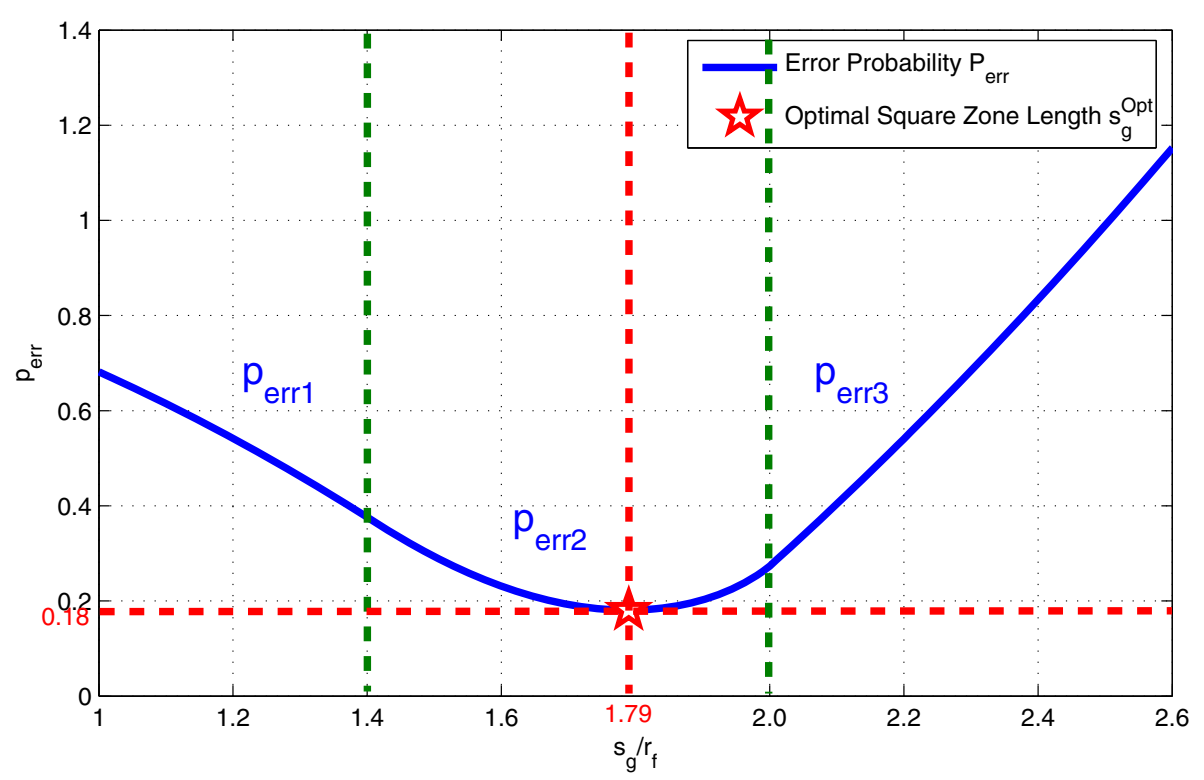

Fig. 11 Minimum error probability $p_{\text {err }}$ and square zone length $s_{g} / r_{f}$ using optimal geographic region division scheme

firstly the error probability of different geographic region division schemes is theoretically analyzed. Then, the optimal region division scheme is proposed and achieved in various cases.

1) Error probability of different geographic region division schemes

The deployment of FBS is modeled as a uniform distribution with the density $\lambda_{\mathrm{FBS}}$, and it is assumed that the coverage of FBS is a circle with a radius of $r_{f}$. The geographic zone is a square with a length of $s_{g}$ and the error probability is denoted by $p_{\text {err }}$ which is shown by the shadow area in Fig. 10. To simplify the theoretical analysis, it is assumed that only one FBS overlaps with the square zone. Then, the error probability $p_{\text {err }}$ is calculated with different $r_{f}$ and $s_{g}$ in three cases below.

Case1: as shown in Fig. 10b, when $0<s_{g} \leq \sqrt{2} r_{f}$, the normalized error probability $p_{\text {err1 }}$ using the geographic region division scheme is depicted in (47).

$$
p_{\mathrm{err} 1}=\left(\pi r_{f}^{2}-s_{g}^{2}\right) / \pi r_{f}^{2}
$$

Case2: as shown in Fig. 10c, when $\sqrt{2} r_{f}<s_{g}<2 r_{f}$, the normalized error probability $p_{\text {err2 }}$ using the geographic region division scheme is depicted in (49), where $\angle \mathrm{AOB}=$ $\alpha=\arccos \left(s_{g} / 2 r_{f}\right), \angle \mathrm{BOC}=\beta=\pi / 4-\alpha, 0<\alpha, \beta<$ $\pi / 4 . S_{\mathrm{OBD}}, S_{\mathrm{OBA}}, S_{\mathrm{OCB}}$, and $S_{\mathrm{OFB}}$ depict the triangle areas.

$$
\begin{aligned}
p_{\mathrm{err} 2}= & 8 / \pi r_{f}^{2}\left[\left(S_{\mathrm{OBD}}-S_{\mathrm{OBA}}\right)+\left(S_{\mathrm{OCB}}-S_{\mathrm{OFB}}\right)\right] \\
= & 8 / \pi r_{f}^{2}\left\{\left(\alpha r_{f}^{2} / 2-\sqrt{4 r_{f}^{2}-s_{g}^{2}} s_{g} / 8\right)\right. \\
& \left.+\left[\left(s_{g}-\sqrt{4 r_{f}^{2}-s_{g}^{2}}\right) s_{g} / 8-\beta r_{f}^{2} / 2\right]\right\} \\
= & 1 / \pi r_{f}^{2}\left[8 r_{f}^{2} \arccos \left(s_{g} / 2 r_{f}\right)-\pi r_{f}^{2}+s_{g}^{2}\right. \\
& \left.-2 s_{g} \sqrt{4 r_{f}^{2}-s_{g}^{2}}\right]
\end{aligned}
$$

Case3: as shown in Fig. 10d, when $s_{g} \geq 2 r_{f}$, the normalized error probability $p_{\text {err3 }}$ using the geographic region division scheme is depicted in (49).

$$
p_{\mathrm{err} 3}=\left(s_{g}^{2}-\pi r_{f}^{2}\right) / \pi r_{f}^{2}
$$

The normalized error probability of dividing geographic region $p_{\text {err }}$ is denoted in (50).

$p_{\text {err }}=\left\{\begin{array}{c}\left(\pi r_{f}^{2}-s_{g}^{2}\right) / \pi r_{f}^{2} \text { when } 0<s_{g} \leq \sqrt{2} r_{f} \\ 8 r_{f}^{2} \arccos \left(\frac{s_{g}}{2 r_{f}}\right)-\pi r_{f}^{2}+s_{g}^{2}-2 s_{g} \sqrt{4 r_{f}^{2}-s_{g}^{2}} / \pi r_{f}^{2} \text { when } \sqrt{2 r_{f}}<s_{g}<2 r_{f} \\ \left(s_{g}^{2}-\pi r_{f}^{2}\right) / \pi r_{f}^{2} \text { when } s_{g} \geq 2 r_{f}\end{array}\right.$

2) Optimal geographic region division scheme

The optimal geographic region division scheme is defined as an optimization problem that how to design an 


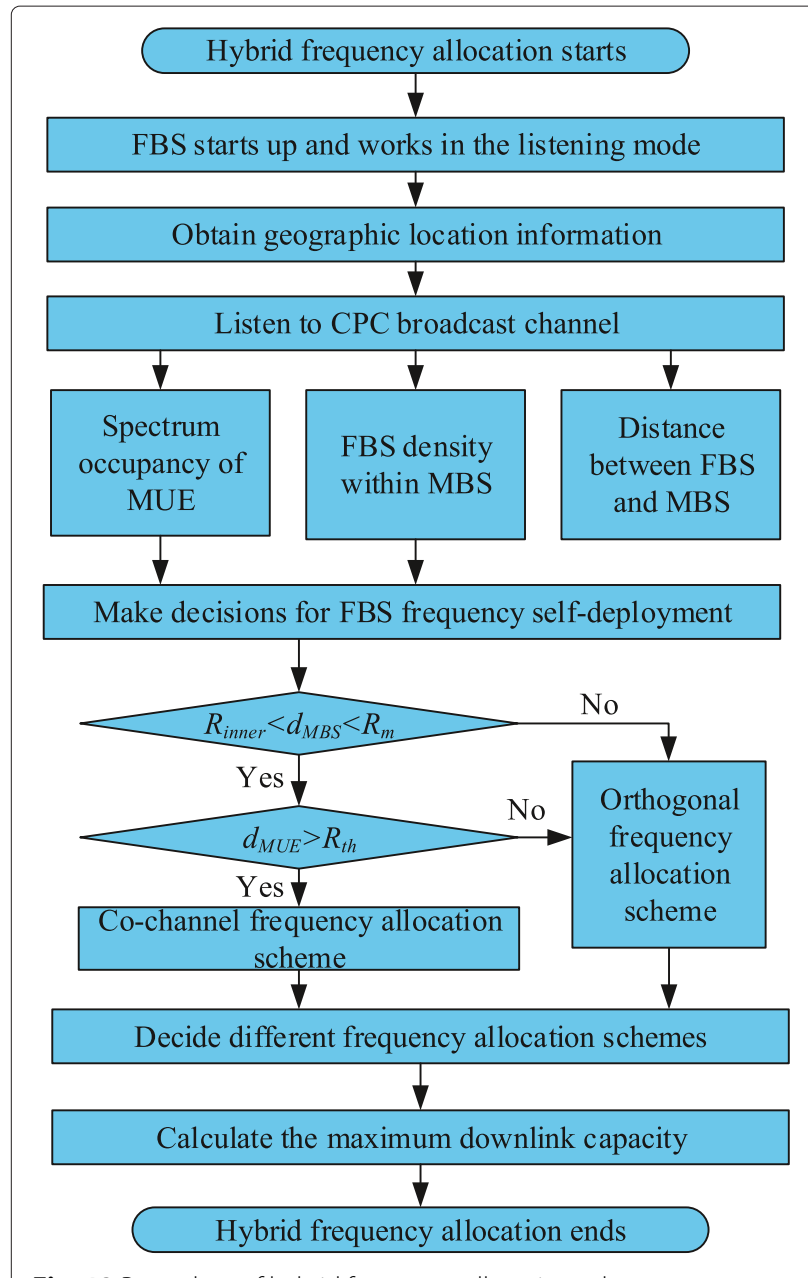

Fig. 12 Procedure of hybrid frequency allocation scheme

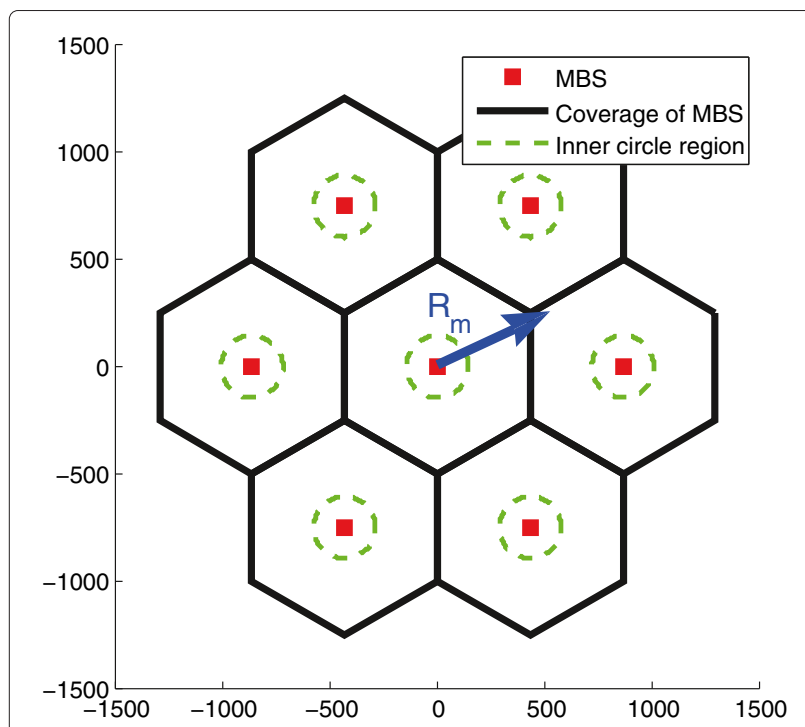

Fig. 13 Simulation scenario optimal square zone length $s_{g}^{\text {Opt }}$ with the minimum error probability $p_{\text {err }}$, as denoted in (51).

$$
s_{g}^{\mathrm{Opt}}=\underset{s_{g}}{\arg \min } p_{\mathrm{err}}
$$

1) When $0<s_{g} \leq \sqrt{2} r_{f}, \quad p_{\text {err1 }}$ is a monotonically decreasing function as denoted in (52). The minimum error probability achieves $p_{\text {err1 }}^{\min }=p_{\text {err1 }}\left(\sqrt{2} r_{f}\right)=\pi-$ $2 / \pi=0.36$, when $s_{g}=\sqrt{2} r_{f}$.

$$
d p_{\mathrm{err} 1} / d s_{g}=-2 s_{g} /\left(\pi r_{f}^{2}\right)<0
$$

2) When $\sqrt{2} r_{f}<s_{g}<2 r_{f}$, by applying $s_{g}=2 r_{f} \cos \alpha$ in (49), $p_{\text {err2 }}$ is depicted in (53), where $\gamma=\arcsin (2 / \sqrt{5})$. When $\alpha=\pi / 2-\arcsin (2 / \sqrt{5}), s_{g}=2 r_{f} \cos \alpha=$ $1.79 r_{f}$, the minimum error probability is $p_{\mathrm{err} 2}^{\min }=$ $[3 \pi-8 \arcsin (2 / \sqrt{5})] / \pi=0.18$.

$$
p_{\text {err } 2}=\frac{1}{\pi}[2-\pi+8 \alpha+2 \sqrt{5} \cos (2 \alpha+\gamma)],
$$

3) When $s_{g} \geq 2 r_{f}, p_{\text {err3 }}$ is a monotonically increasing function as denoted in (54). The minimum error probability achieves $p_{\mathrm{err} 3}^{\min }=p_{\mathrm{err} 3}\left(2 r_{f}\right)=(4-\pi) / \pi=0.27$, when $s_{g}=2 r_{f}$.

$$
d p_{\mathrm{err} 3} / d s_{g}=2 s_{g} /\left(\pi r_{f}^{2}\right)>0
$$

Therefore, the minimum error probability using geographic region division scheme achieves $p_{\mathrm{err}}^{\min }=$ $\min \left(p_{\mathrm{err} 1}^{\min }, p_{\mathrm{err} 2}^{\min }, p_{\mathrm{err} 3}^{\min }\right)=p_{\mathrm{err} 2}^{\min }=0.18$, when the optimal square zone length $s_{g}^{\text {Opt }}=\underset{s_{g}}{\arg \min } p_{\text {err }}=1.79 r_{f}$. In addition, under different geographic region division ratio $s_{g} / r_{f}$ conditions, the trend of error probability $p_{\text {err }}$ is depicted in Fig. 11. Then, the minimum error probability achieves

Table 1 Simulation parameters

\begin{tabular}{ll}
\hline Parameters & Value \\
\hline Transmit power of $M B S P_{m}(\mathrm{~W})$ & 20 \\
Transmit power of FBS $P_{f}(\mathrm{~W})$ & 0.1 \\
System bandwidth $B W(\mathrm{MHz})$ & 20 \\
RB number $N$ & 110 \\
Path loss $\alpha$ & 4 \\
Target SINR (dB) & 3 \\
Maximum outage probability $\epsilon$ & 0.1 \\
Coverage radius of MBS $R_{m}(\mathrm{~m})$ & 500 \\
Coverage radius of FBS $R_{f}(\mathrm{~m})$ & 10 \\
Background noise power $N_{0}(\mathrm{~W})$ & $10^{-12}$ \\
Inner circle region radius $R_{\text {inner }}(\mathrm{m})$ & 59.5 \\
Threshold radius $R_{\text {th }}(\mathrm{m})$ & $10,20,30,40,50$
\end{tabular}


Orthogonal Scheme $\rho=0.9$
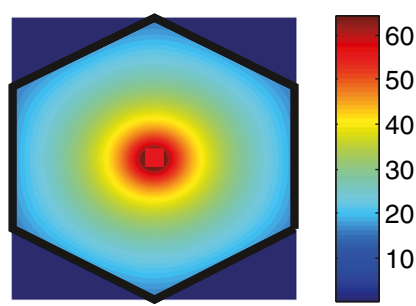

Hybrid Scheme $\lambda_{\text {FBS }} 2=9.7975^{*} 10^{-7}$

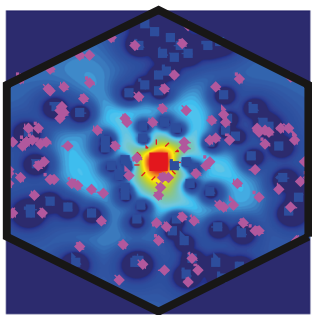

Hybrid Scheme $\lambda_{\mathrm{FBS}}{ }^{1=4.1989^{*} 10^{-7}}$
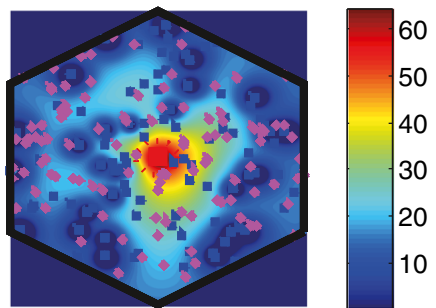

Hybrid Scheme $\lambda_{\mathrm{FBS}} 3=1.5396^{*} 10^{-6}$
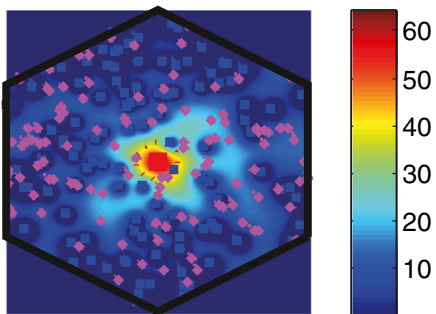

Fig. 14 SINR of MUE with different FBS density $\lambda_{\text {FBS }}$

$p_{\mathrm{err}}^{\min }=0.18$ and the optimal square zone length is $s_{g}^{\text {Opt }}=$ $1.79 r_{f}$.

\section{Self-deployment procedure of hybrid frequency allocation scheme}

In order to efficiently deliver the frequency occupancy information of MBS in different geographic zones, the in-band CPC technology is applied as a candidate solution in [22]. Therefore, the self-deployment procedure of FBS using hybrid frequency allocation scheme is shown in Fig. 12.
Step 1: FBS starts up and works in the listening mode. And FBS receives the frequency occupancy information of MBS via the CPC channel and exchanges the selfdeployment default parameters with the core network via backhaul links.

Step 2: Second, FBS monitors the CPC and depicts key parameters of heterogeneous networks, such as the frequency occupancy information of MUE within the vicinity of FBS, the density of FBS, the received signal strength of MBS to estimate the distance between FBS and MBS.

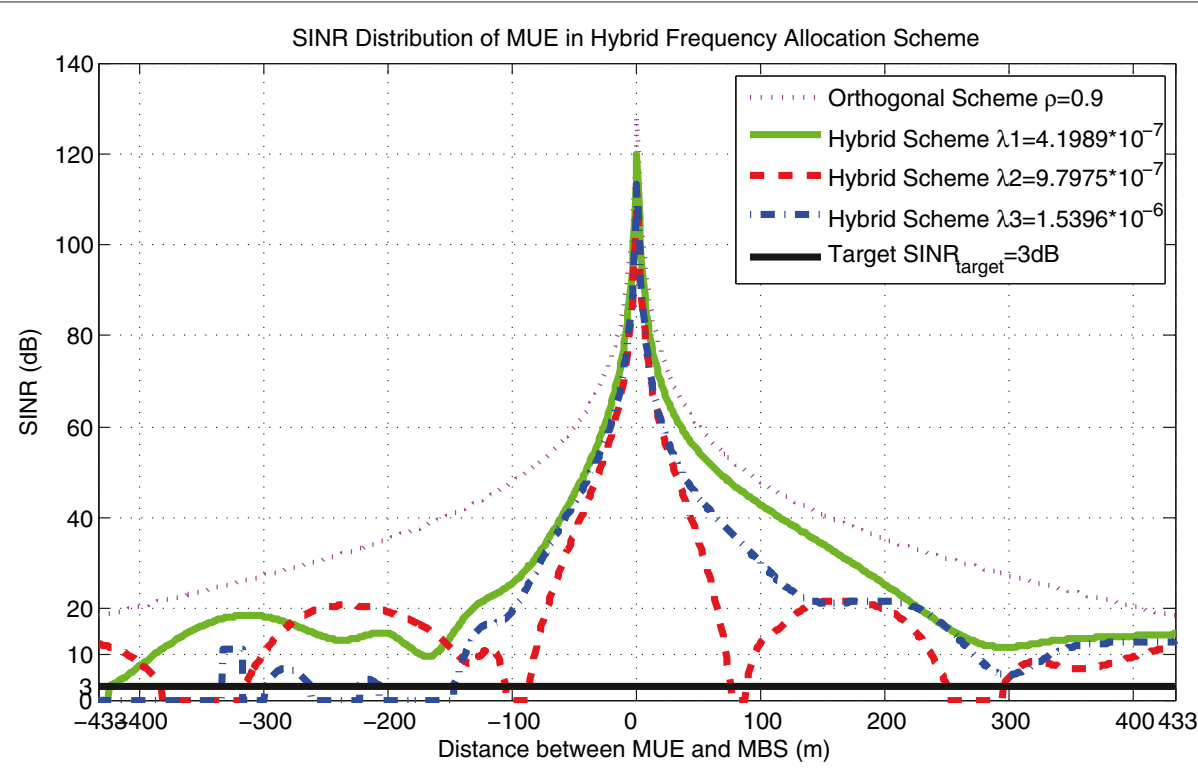

Fig. 15 SINR distribution of MUE with different distances between MUE and MBS 

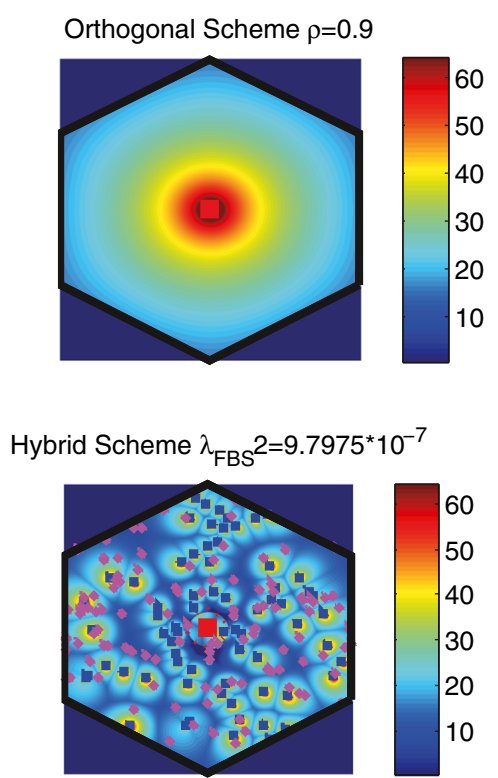

Fig. 16 SINR of FUE with different FBS density $\lambda_{F B S}$

Step 3: FBS makes spectrum self-deployment decisions based on the calculation of $R_{\mathrm{inner}}$ and $R_{\mathrm{th}}$. By comparing the distance of $d_{\mathrm{MBS}}$ to $R_{\text {inner }}$, FBS can decide whether it belongs to the inner circle region or the outer circle region. Then, FBS makes a decision on applying different frequency allocation schemes, such as orthogonal, cochannel, and hybrid frequency allocation schemes, based on the distance between $d_{\mathrm{MUE}}$ and $R_{\mathrm{th}}$.
Hybrid Scheme $\lambda_{\mathrm{FBS}}{ }^{1=4.1989^{*} 10^{-7}}$

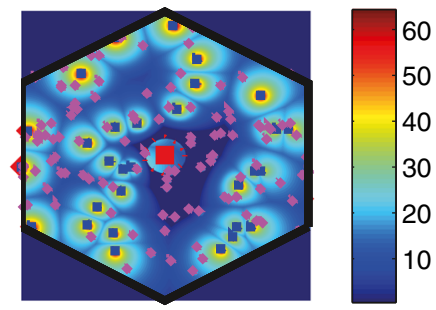

Hybrid Scheme $\lambda_{\mathrm{FBS}}{ }^{3=1.5396 * 10^{-6}}$
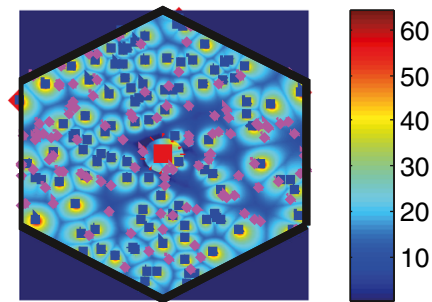

Step 4: The maximum downlink capacity of heterogeneous network is calculated and the procedure of hybrid frequency allocation scheme ends.

\section{Numerical results and analysis}

In this section, numerical results of SINR and capacity of FBS and MBS are described by applying the proposed hybrid frequency allocation scheme. A typical scenario is

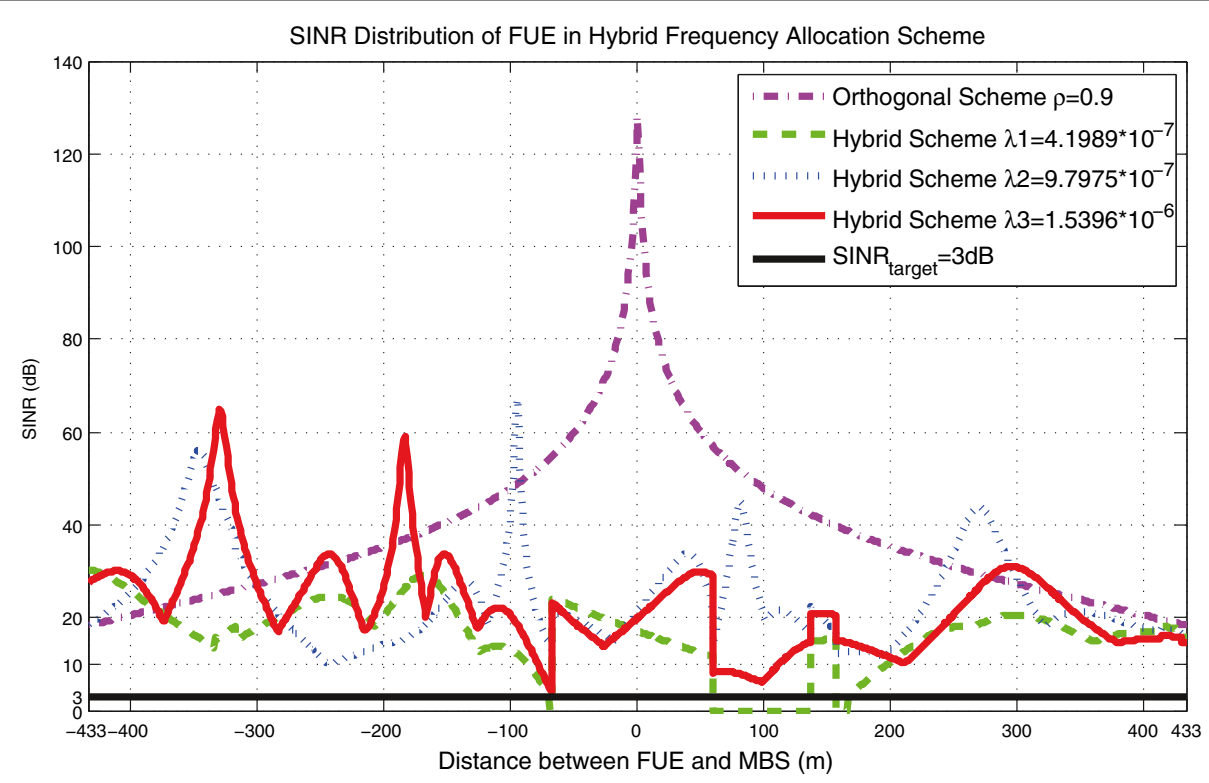

Fig. 17 SINR distribution of FUE with different distances between FUE and MBS 


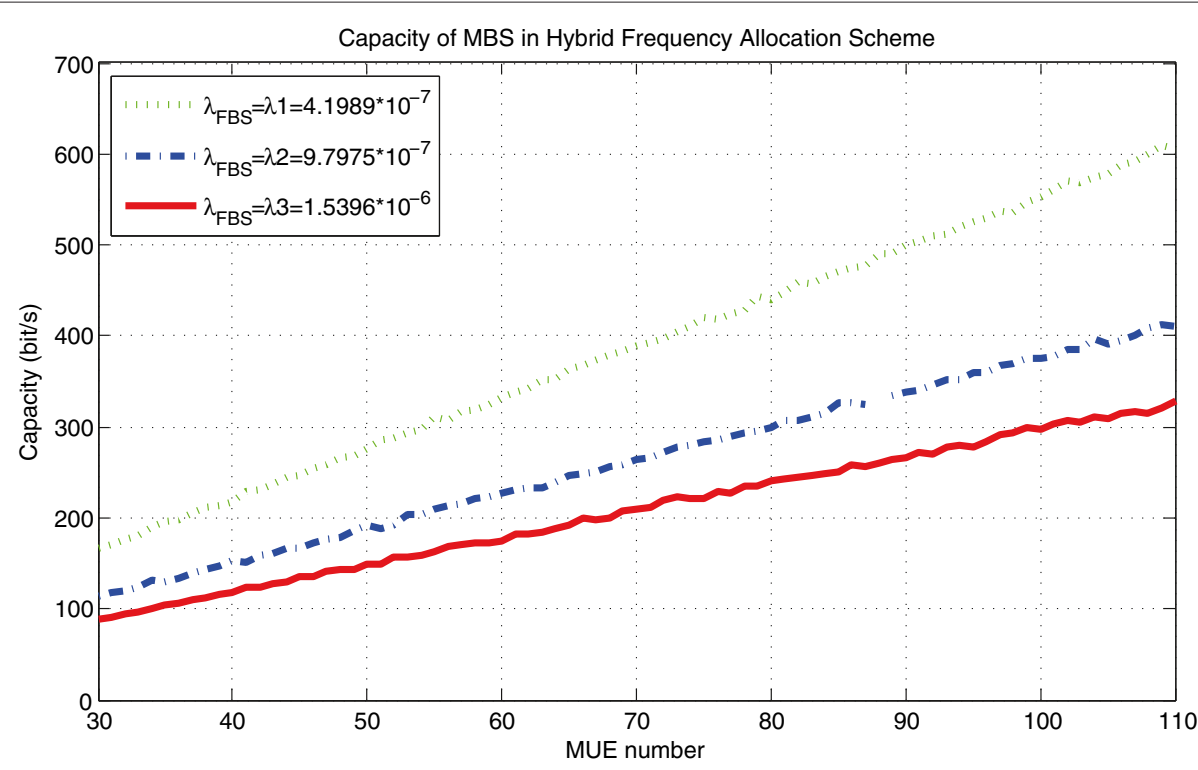

Fig. 18 Capacity of MBS with different FBS density and MUE density

depicted in Fig. 13, which includes seven MBSs and the proposed outer and inner circle regions. Key parameters in the typical simulation scenario are denoted in Table 1 [28]. Simulation results of various SINR and capacity values with different FBS densities and distances between MBS and FBS are analyzed below using the proposed hybrid frequency allocation scheme.

\subsection{SINR analysis of hybrid frequency allocation scheme} The SINR distribution of MUE in different locations in the hybrid frequency allocation scheme is shown in Fig. 14, where the warmer color denotes a much higher SINR value. MUE in the outer circle region will suffer the strong interference from neighboring MBSs and FBSs outside a distance of $R_{\mathrm{th}}$, which will greatly increase with the surge of FBS density $\lambda_{\mathrm{FBS}}$ from $\lambda_{1}$ to $\lambda_{3}$. Furthermore, the SINR distribution of MUE is also depicted in Fig. 15 which demonstrates the effect of the distance between MUE and MBS to SINR values. Two curves of the orthogonal frequency allocation scheme and the target SINR value are also plotted in Fig. 15 as references. The SINR value of MUE achieves a small peak at a distance around

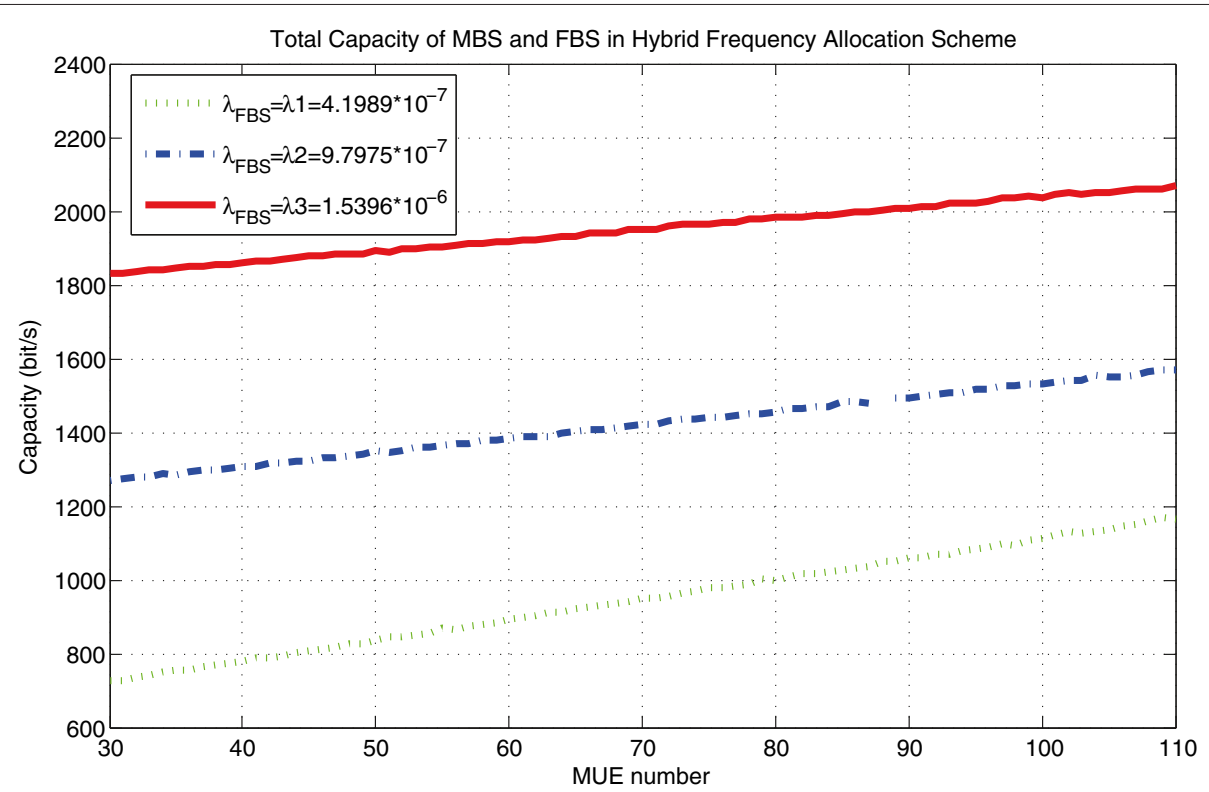

Fig. 19 Total capacity of MBS and FBS with different FBS density and MUE density 


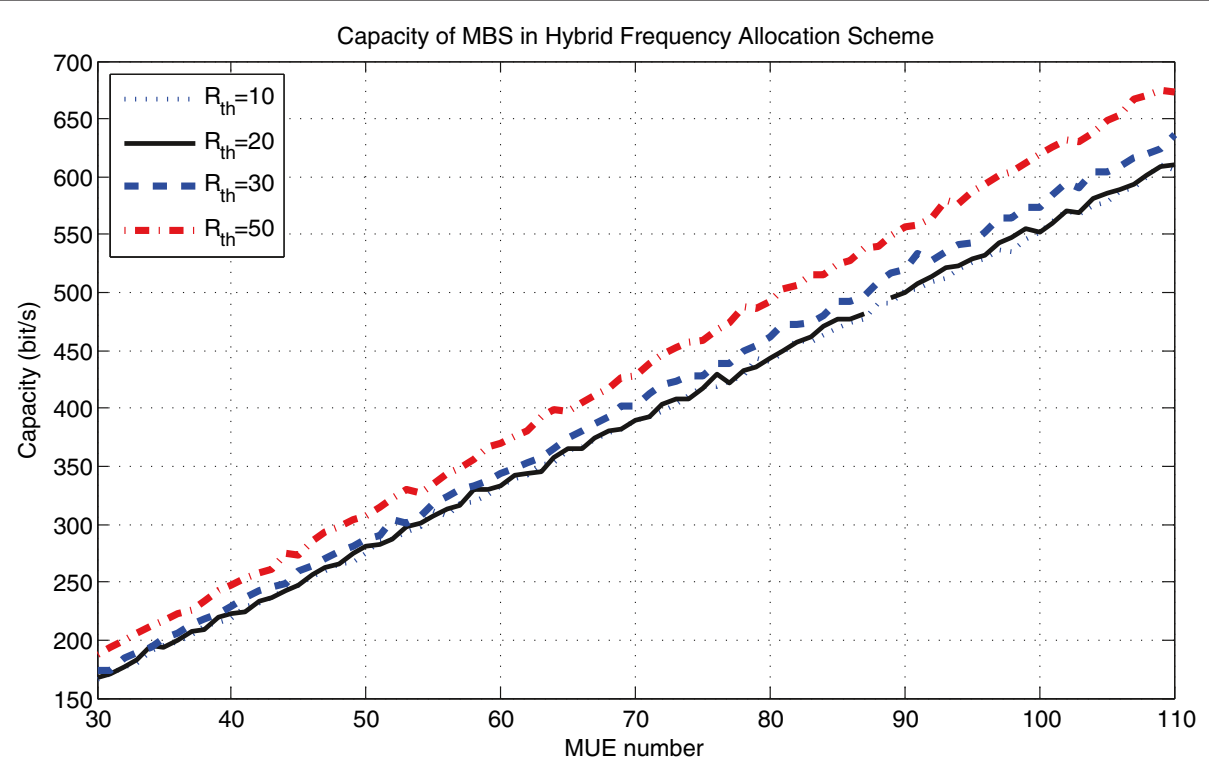

Fig. 20 Capacity of MBS with different $R_{\text {th }}$

$200 \mathrm{~m}$ away from MBS, which denotes that the orthogonal frequency allocation scheme is applied to FBS within a distance of $R_{\text {th }}$ of MUE. With the increase of FBS density $\lambda_{\text {FBS }}$, the SINR value of MUE will decrease extensively which denotes that MUE suffers from the strong interference from neighboring FBSs outside a distance of $R_{\text {th }}$. Therefore, MUE on the edge of MBS can dynamically increase the threshold radius $R_{\text {th }}$ to decrease the co-channel interference from densely deployed FBSs.
Moreover, the SINR distribution of FUE is shown in Fig. 16 with different FBS density values. With the increase of FBS, the SINR of FUE within FBS's coverage increases. But when the density of FBS is extensively large, the SINR of FUE will decrease due to the strong interference from neighboring FBSs around the FUE. Thus, the SINR distribution of FUE is also depicted in Fig. 17 which demonstrates the effect of the distance between FUE and MBS to SINR values. Two curves of the orthogonal

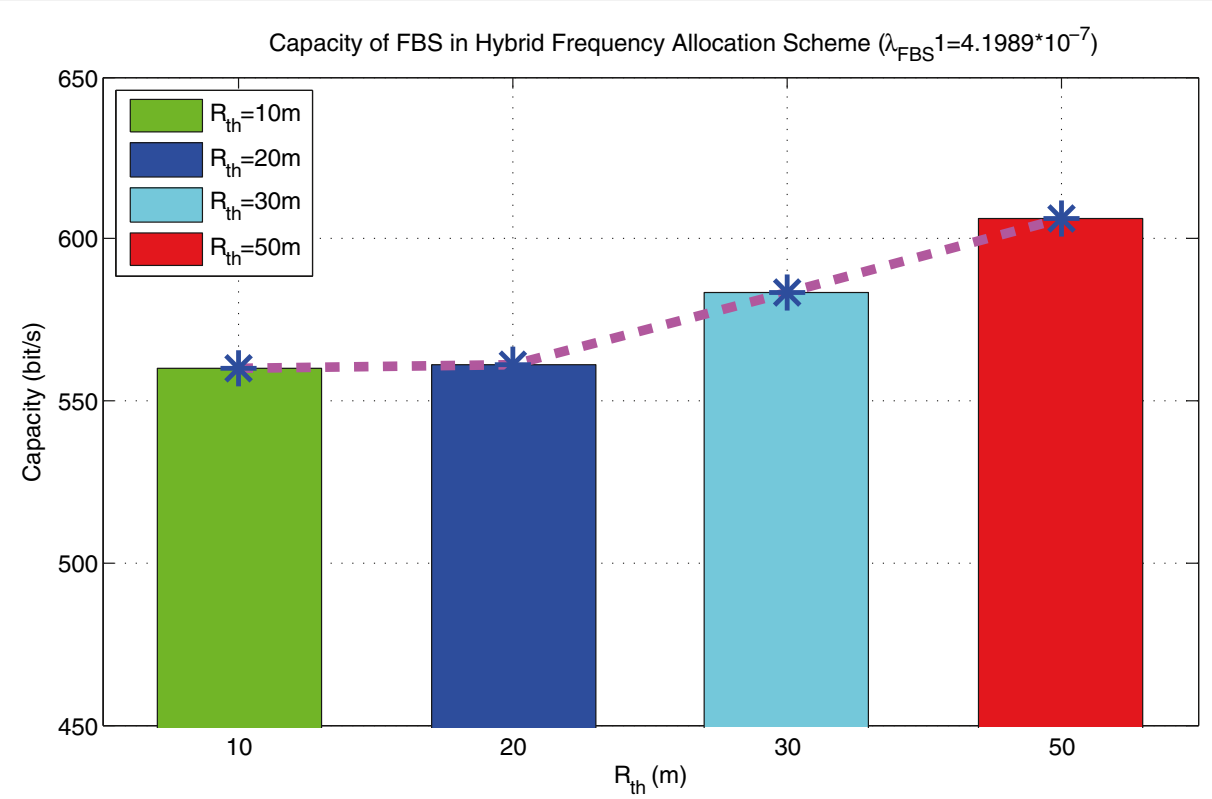

Fig. 21 Capacity of FBS with different $R_{\text {th }}$ when $\lambda_{\mathrm{FBS}}=4.1989 \times 10^{-7}$ 
frequency allocation scheme and the target SINR value are also plotted in Fig. 17 as references. The SINR value of FUE achieves several small peaks at the distance around 200 and $300 \mathrm{~m}$ away from MBS, which denotes that the orthogonal frequency allocation scheme is applied to neighboring FBSs to decrease co-channel interferences. Furthermore, the appropriate increase of $R_{\text {th }}$ can also decrease the co-channel interference to FUE from neighboring FBSs, especially when FBS density $\lambda_{\mathrm{FBS}}$ is large.

\subsection{Capacity analysis of hybrid frequency allocation scheme}

As shown in Fig. 18, the capacity of MBS increases with the increase of MUE density. Due to the co-channel interference from densely deployed neighboring FBSs, the capacity of MBS decreases with the increase of FBS den-

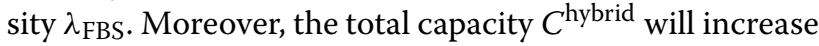
with the surge of FBS density $\lambda_{\text {FBS }}$ as shown in Fig. 19, which depicts that the densely deployed FBSs can increase the total capacity with appropriate $R_{\text {th }}$ to minimize the co-channel interference. And the total capacity will also increase with the increase of MUE density. Moreover, the co-channel interference from neighboring FBS to MUE can be minimized and the capacity of MBS and FBS will also increase with the increase of $R_{\text {th }}$ as shown in Figs. 20 and 21.

\section{Conclusions}

To increase the downlink capacity of hierarchical networks, this paper proposes a hybrid frequency allocation scheme with densely deployed small cells. First, based on the theoretical modeling and mathematical analysis, three frequency allocation schemes are proposed and key parameters affecting the capacity are defined and proved with closed-form solutions in different scenarios. And the outer and inner circle regions are proposed to minimize the co-channel interference to MUE from neighboring FBS. Second, the optimal geographic region division scheme is proposed with optimal square zone length solutions, which can improve the information delivery among small cells. Furthermore, the self-deployment procedure of proposed hybrid frequency allocation scheme is also designed in this paper. Finally, numerical results are presented and analyzed to verify the capacity improvement of proposed scheme by considering the density of FBS, co-channel interference and the spatial separation among small cells.

\section{Competing interests}

The authors declare that they have no competing interests.

\section{Acknowledgments}

The project was supported by the Key Laboratory of Universal Wireless Communications (KFKT-2013103), the Fundamental Research Funds for the Central Universities (2014ZD03-02), the Beijing Municipal Science and Technology Commission research fund project (D151100000115002), the
National Natural Science Foundation of China (61201152), and the National High-Tech R\&D Program (863 Program 2015AA01A705).

Received: 18 July 2014 Accepted: 14 May 2015

Published online: 04 June 2015

\section{References}

1. G Mansfield, Femtocells in the US market-business drivers and consumer propositions. FemtoCells Europe, 1927-1948 (2008)

2. J Zhang, G de la Roche, Femtocells: Technologies and Deployment. (John Wiley \& Sons Ltd, UK, 2010), pp. 1-13

3. J Cullen, Radioframe presentation, (Femtocell Europe, London, UK, 2008)

4. VNI Cisco Forecast white paper (2015). http://www.cisco.com/c/en/us/ solutions/collateral/service-provider/visual-networking-index-vni/ white_paper_c11-520862.pdf

5. W Webb, Wireless Communications: The Future. (John Wiley \& Sons, UK, 2007)

6. V Chandrasekhar, JG Andrews, A Gatherer, Femtocell network: a survey. IEEE Commun. Mag. 46(9), 59-67 (2008)

7. JG Andrews, H Claussen, M Dohler, S Rangan, Femtocells: past, present, and future. IEEE J. Sel. Areas Commun. 30(3), 497-508 (2012)

8. P Xia, V Chandrasekhar, JG Andrews, Femtocell access control in the TDMANOFDMA uplink, (Miami, USA, 6-10 Dec. 2010)

9. H ElSawy, E Hossain, DI Kim, HetNets with cognitive small cells: user offloading and distributed channel access techniques. IEEE Commun. Mag. $\mathbf{5 1}(6), 28-36(2013)$

10. D Mavrakis, Small Cell Market Status. (Informa Telecoms \& Media, 2013)

11. P Xia, V Chandrasekhar, JG Andrews, Open vs. closed access femtocells in the uplink. IEEE Trans. Wireless Commun. 9(12), 3798-3809 (2010)

12. V Chandrasekhar, JG Andrews, Spectrum allocation in tiered cellular networks. IEEE Trans. Commun. 57(10), 3059-3068 (2009)

13. CC Wang, TQS Quek, M Kountouris, Throughput optimization, spectrum allocation, and access control in two-tier femtocell networks. IEEE J. Sel. Areas Commun. 30(3), 561-574 (2012)

14. I Guvenc, M-R Jeong, F Watanabe, H Inamura, A hybrid frequency assignment for femtocells and coverage area analysis for co-channel operation. IEEE Commun. Lett. 12(12), 880-882 (2008)

15. A Galindo-Serrano, L Guipponi, M Dohler, in IEEE Global Telecommunications Conference (GLOBECOM). Cognition and docition in OFDMA-based femtocell networks (Miami, USA, 6-10 Dec. 2010)

16. V Chandrasekhar, JG Andrews, Uplink capacity and interference avoidance for two-tier femtocell networks. IEEE Trans. Wireless Commun. 8(7), 3498-3509 (2009)

17. D Lopez-Perez, A Valcarce, G de la Roche, J Zhang, OFDMA femtocells: a roadmap on interference avoidance. IEEE Commun. Mag. 47(9), 41-48 (2009)

18. D Lopez-Perez, A Ladanyi, A Juttner, J Zhang, in IEEE International Symposium on Personal Indoor and Mobile Radio Communications (PIMRC). OFDMA femtocells: A self-organizing approach for frequency assignment (Tokyo, Japan, 13-16 Sept. 2009)

19. S Lien, C Tseng, K Chen, C Su, in IEEE International Conference on Communications (ICC). Cognitive radio resource management for QoS guarantees in autonomous femtocell networks (Cape Town, South Africa, p. 23-27 May 2010

20. H ElSawy, E Hossain, Two-tier HetNets with cognitive femtocells: downlink performance modeling and analysis in a multichannel environment. IEEE Trans. Mobile Comput. 13(3), 649-663 (2014)

21. CHM Lima, M Bennis, M Latva-aho, Coordination mechanisms for self-organizing femtocells in two-tier coexistence scenarios. IEEE Trans. Wireless Commun. 11(6), 2212-2223 (2012)

22. Q Zhang, Z Feng, G Zhang, Q Li, P Zhang, Efficient mesh division and differential information coding schemes in broadcast cognitive pilot channel. Wireless Pers. Commun. 63(2), 363-392 (2012)

23. K Nolte, A Kaloxylos, K Tsagkaris, T Rosowski, M Stamatelatos, A Galani, E Bogenfeld, P Magdalinos, J Tiemann, J Gebert, P Arnold, D Hugo, N Alonistioti, P Demestichas, W Koenig, The E3 architecture: enabling future cellular networks with cognitive and self-x capabilities. Int. J. Netw. Manag 21, 1-24 (2010). doi: 10.1002/nem.762.

24. S-M Cheng, WC Ao, K-C Chen, in IEEE International Symposium on Personal Indoor and Mobile Radio Communications (PIMRC). Downlink capacity of two-tier cognitive femto networks Istanbul, Turkey, 26-30 Sept. 2010)

25. J Kingman, Poisson Precesses. (Oxford University Press, Oxford, 1993) 
26. Poisson distribution, Wikipedia. http://en.wikipedia.org/wiki/ Poisson_distribution\#2-dimensional_Poisson_process.

27. F Baccelli, B Blaszczyszyn, P Muhlethaler, An aloha protocol for multihop mobile wireless networks. IEEE Trans. Inf. Theory. 52(2), 421-436 (2006)

28. 3GPP TR 36.814 V9.0.0, 3GPP TSG RAN (E-UTRA): further advancements for E-UTRA physical layer aspects (Release 9) (2010)

Submit your manuscript to a SpringerOpen ${ }^{\circ}$ journal and benefit from:

- Convenient online submission

Rigorous peer review

- Immediate publication on acceptance

- Open access: articles freely available online

- High visibility within the field

- Retaining the copyright to your article

Submit your next manuscript at $\boldsymbol{\triangleright}$ springeropen.com 